\title{
Crystal structure of the human NKR-P1 bound to its lymphocyte ligand LLT1 reveals receptor clustering in the immune synapse
}

Jan Bláha ${ }^{1, \#}$, Tereza Skálová ${ }^{2}$, Barbora Kalousková ${ }^{1}$, Ondřej Skořepa ${ }^{1}$, Denis Cmunt $^{1, \S}$, Samuel Pažický1,\#, Edita Poláchová ${ }^{1}$, Celeste Abreu ${ }^{1}$, Jan Stránský², Tomáš Koval'², Jarmila Dušková2 ${ }^{2}$ Yuguang Zhao ${ }^{3}$, Karl Harlos ${ }^{3}$, Jindřich Hašek², Jan Dohnálek², Ondřej Vaněk ${ }^{1, *}$

${ }^{1}$ Department of Biochemistry, Faculty of Science, Charles University, Hlavova 2030, 12840 Prague, Czech Republic

2 Institute of Biotechnology, The Czech Academy of Sciences, BIOCEV Centre, Průmyslová 595, 25250 Vestec, Czech Republic

${ }^{3}$ Division of Structural Biology, The Wellcome Trust Centre for Human Genetics, University of Oxford, Roosevelt Drive, OX3 7BN Oxford, United Kingdom

\# Present address: EMBL, Hamburg Unit c/o DESY, Notkestrasse 85, 22607 Hamburg, Germany

$\S$ Present address: Department of Oncology, Ludwig Institute for Cancer Research, University of Lausanne, Chemin des Boveresses 155, 1066 Epalinges, Switzerland

* Corresponding author. Tel.: +420221951272, E-mail: ondrej.vanek@natur.cuni.cz

\begin{abstract}
Human NKR-P1 (CD161, KLRB1) and its ligand LLT1 (CLEC2D) are a prototypical inhibitory C-type lectin-like receptor:ligand pair of NK cells with a critical role in homing lymphocytes to immune-privileged sites, particularly in multiple sclerosis, rheumatoid arthritis, and Crohn's disease. Furthermore, NKR-P1:LLT1 inhibitory signaling is associated with glioblastoma, non-Hodgkin's lymphoma, breast, and prostate cancer. However, the lack of structural data on the formation of the NKR-P1:LLT1 complex limits our understanding of this signaling. We thus solved the crystal structures of NKR-P1 and the NKR-P1:LLT1 complex. NKR-P1 forms a homodimer with an unexpected arrangement that enables LLT1 binding in two modes, bridging two LLT1 molecules, thereby forming interaction clusters suggestive of an inhibitory immune synapse. Moreover, observing the formation of these clusters by SEC-SAXS analysis in solution and by dSTORM super-resolution microscopy on the cell surface, and following their role in receptor signaling using in vitro cytotoxicity assay with freshly isolated NK cells, we show how NKR-P1:LLT1 clustering allows these proteins to overcome the weak affinity of C-type lectin-like receptors to their ligands. Furthermore, only the ligation of both primary and secondary LLT1 binding interfaces leads to effective NKR-P1 inhibitory signaling. Therefore, our findings show how inhibitory receptor cross-linking and clustering work together to trigger signal transduction upon cellular contact in the immune synapse.
\end{abstract}


Keywords: NKR-P1; CD161; KLRB1; LLT1; CLEC2D; NK cells; C-type lectin-like; X-ray structure; SEC-SAXS; dSTORM

\section{Introduction}

Natural killer (NK) cells are innate immune lymphocytes equipped with a wide range of activating and inhibitory surface receptors, allowing them to sensitively recognize and kill malignant, infected, or other transformed cells through "missing-" and "induced-self" mechanisms and through antibody-dependent cell-mediated cytotoxicity $(A D C C)^{1}$. In addition, NK cells also contribute to the initiation and development of the adaptive immune response, secreting several classes of cytokines, especially proinflammatory IFN- $\gamma^{1}$. Interestingly, recent findings show that NK cells are even capable of maintaining a form of immunological memory ${ }^{1,2}$, thus further highlighting the principal roles NK cells play in immunity, particularly through their receptors.

NK receptors comprise two structurally divergent classes: the families of immunoglobulin-like receptors and C-type lectin-like receptors (CTLRs) ${ }^{3,4}$. CTLRs are encoded within the natural killer gene complex (NKC, human chromosome 12), and, unlike C-type lectins, CTLRs neither bind calcium ions nor engage carbohydrate ligands ${ }^{5,6}$. Instead, CTLRs are known to interact with protein ligands. For example, receptors such as Ly49, CD94/NKG2, or NKG2D recognize MHC class-I like molecules ${ }^{3}$, whereas receptors of the NKR-P1 subfamily recognize structurally highly related Clr/Ocil CTLRs. These are encoded by CLEC2 genes ${ }^{3,5}$ that are genetically tightly linked with the NKR-P1-coding KLR genes. This unique CTLR:CTLR interaction system is involved in both non-MHC missing-self and induced-self recognition ${ }^{3,4,5}$. Several inhibitory and activating NKR-P1 receptors have been described in mice and rats; however, the human receptor NKR-P1 (CD161, KLRB1 gene) remains since 1994 the only human orthologue described so far ${ }^{7}$. Nevertheless, based on structural and functional homology to NKR-P1, the human activating CTLR:ligand pairs NKp65:KACL $(\text { KLRF2:CLEC2A) })^{8}$ and NKp80:AICL (KLRF1:CLEC2B) ${ }^{9}$ have been proposed as the activating counterparts of human NKR-P1 ${ }^{4,10}$.

Human NKR-P1 (CD161) was first reported as a marker of NK cells ${ }^{7}$, in which NKR-P1 acts as an inhibitory receptor ${ }^{7,11,12}$ up-regulated by IL-12 ${ }^{13}$. However, NKR-P1 is also expressed by natural killer $\mathrm{T}$ (NKT) cells ${ }^{14}$, mucosal-associated invariant $\mathrm{T}$ (MAIT) cells ${ }^{15}$, and other subsets of T-lymphocytes ${ }^{16}$, wherein NKR-P1 acts as a co-stimulatory receptor, increasing IFN-y secretion ${ }^{11,17}$. Unsurprisingly, NKR-P1 is even detected in immature CD16- CD56- NK cells $^{18}$ and in precursors of Th17 and MAIT cells in the umbilical cord blood ${ }^{19}$. Recently, NKR-P1 was identified in glioma-infiltrating T cells, having an inhibitory, immunosuppressive role in $\mathrm{T}$ cell-mediated killing of glioma cells $^{20}$. In addition, NKR-P1 promotes 
transendothelial migration to immunologically privileged niches upon interaction with its endogenous ligand, lectin-like transcript 1 (LLT1) ${ }^{19,21,22 .}$

LLT1 (gene CLEC2D) is primarily expressed on activated monocytes and B cells ${ }^{23}$. In these cells, LLT1 helps to maintain NK cell self-tolerance ${ }^{10,23}$. However, IL-2 can induce its expression on NK and T cells ${ }^{24}$. Furthermore, LLT1 is up-regulated on glioblastoma ${ }^{25}$, prostate and triple-negative breast cance ${ }^{26,27}$, and B cell non-Hodgkin's lymphoma ${ }^{28}$ cells, in which LLT1 contributes to immune evasion by dampening NK cell cytotoxicity. Interestingly, increased numbers of $\mathrm{CD}_{161^{+}}$Th17 cells have been detected in glioma tumors ${ }^{29}$. Concomitantly, the functions of NKR-P1 receptors on IL-17-producing regulatory $\mathrm{T}_{\text {cells }}{ }^{30}$, on subsets of Tc17 cells ${ }^{31}$, and all Th17 cells ${ }^{19}$ are particularly relevant because these cells have been implicated in several autoimmune diseases (Crohn's disease ${ }^{32}$, multiple sclerosis $^{33}$, rheumatoid arthritis ${ }^{34}$, and psoriasis $^{35}$ ). Therefore, the analysis of NKR-P1 receptors and ligands such as LLT1 is essential to gain a deeper insight into the structurefunction relationships underlying both physiological and pathogenic processes in the immune system.

Human NKR-P1 and LLT1 are type II transmembrane glycoproteins with similar protein topology4: an N-terminal cytoplasmic signaling tail, a transmembrane helix, a flexible stalk region, and a C-terminal C-type lectin-like domain (CTLD) ${ }^{3,7,36}$. Moreover, both NKR-P1 and LLT1 were shown to form disulfide homodimers ${ }^{7}, 36$, likely linked in their stalk regions. However, the structure of NKp65:KACL complex ${ }^{37}$ is the only one among all complexes of the human CTL receptor:ligand subfamily that has been solved so far. A subsequent study further showed that the interaction between NKp65 and KACL is protein-based and independent of glycosylation ${ }^{38}$. Based on these data, a model of the NKR-P1:LLT1 complex was subsequently proposed, and key interaction residues were identified through surface plasmon resonance (SPR) analysis of NKR-P1 and LLT1 mutants, highlighting the fast kinetics of this interaction 39,40 . Furthermore, the structures of related mouse NKR-P1B ectodomain complexed with murine cytomegalovirus (MCMV) immunoevasin protein m12, or with its cognate ligand Clrb, have been recently reported ${ }^{41,42}$. Notwithstanding, no comprehensive model of the CTLR:ligand complexes' dimer:dimer interaction, corresponding to the natural state of these proteins when expressed on the cells' surface, is available yet.

Previously, we reported the first structure of $\mathrm{LLT1}^{43}$ forming a non-covalent dimer following the conserved dimerization mode of CLEC2-encoded ligands, regardless of glycosylation ${ }^{44}$. Here, we investigated the structure of human NKR-P1 and examined the effects of NKR-P1 dimerization on LLT1 binding, thereby solving the structure of the human NKR-P1 receptor. Accordingly, in this study, we show that NKR-P1 forms a non-covalent dimer that differs from the dimerization mode observed for $\mathrm{CD} 69^{45,46}, \mathrm{Clrg}^{47}$, and LLT1 ${ }^{43}$, and we discuss the role of glycosylation in its dimerization. Furthermore, the crystal structure of NKR-P1 in complex 
with LLT1 explains the previous in-solution interaction observations while simultaneously showing a novel assembly of this complex utilizing two different non-symmetric binding sites on LLT1. Lastly, by combining dSTORM nanoscopy cluster analysis of NKR-P1 on the cell surface with data from the obtained crystal structures, biophysical interaction and structural analyses in solution, and in vitro NK cell killing assays, we show for the first time that human NKR-P1 receptor overcomes its weak affinity for LLT1 by ligand binding-induced crosslinking and clustering, thus elucidating the mode of signal transduction of this receptor within the NK cell immune synapse.

\section{Results}

\section{Structure of the human NKR-P1 ectodomain}

Two crystal structures of the human NKR-P1 ectodomain were solved: the structure of glycosylated NKR-P1 possessing uniform Asn-GlcNAc $2 \mathrm{Man}_{5}$ N-glycans (NKR-P1_glyco) and of deglycosylated NKR-P1 with N-glycans cleaved off after the first GlcNAc residue (NKRP1_deglyco); statistical data on all structures are outlined in Table 1. NKR-P1 in both crystal structures follows the general fold characteristic of a CTL domain - two $\alpha$-helices ( $\alpha 1$ and $\alpha 2)$ and two antiparallel $\beta$-sheets with the conserved hydrophobic WIGL motif within the domain core (Figs. 1 and $2 a$ ). The two $\beta$-sheets are formed by $\beta$-strands $\beta 0, \beta 1, \beta 1$ ' and $\beta 5$, and $\beta 2, \beta 2$ ', $\beta 3$, and $\beta 4$, respectively (assignment according to Zelensky and Gready ${ }^{5}$, also used to describe other related CTL structures ${ }^{37,40}$ ). In addition, three intramolecular disulfide bonds stabilize the domain: Cys94-Cys105, Cys122-Cys210, and Cys189-Cys202.

Table 1. Data processing statistics and structure refinement parameters. Values in parentheses refer to the highest resolution shell.

\begin{tabular}{|c|c|c|c|}
\hline Crystal structure & NKR-P1 glyco & NKR-P1 deglyco & NKR-P1:LLT1 \\
\hline PDB code & 5MGR & $5 \mathrm{MGS}$ & 5MGT \\
\hline \multicolumn{4}{|c|}{ Data processing statistics } \\
\hline Space group & $P 3_{1} 21$ & P1 & $\mathrm{P} 2{ }_{1} 2_{1} 2_{1}$ \\
\hline $\begin{array}{l}\text { Unit-cell parameters } a, b, c(A) ; \alpha, \\
\beta, Y\left(^{\circ}\right)\end{array}$ & $\begin{array}{l}68.24,68.24,127.19 \\
90,90,120\end{array}$ & $\begin{array}{c}44.81,68.40,101.56 \\
101.88,100.72,100.64\end{array}$ & $\begin{array}{c}44.58,80.15,272.95 ; 90 \\
90,90\end{array}$ \\
\hline Resolution range $(\AA)$ & $\begin{array}{c}43.3-1.8(1.84- \\
1.80)\end{array}$ & $\begin{array}{l}48.68-1.90 \\
(1.93-1.90)\end{array}$ & $76.90-1.90(1.94-1.90)$ \\
\hline No. of observations & $1287150(76917)$ & $610421(26224)$ & $1452154(80494)$ \\
\hline No. of unique reflections & 32555 (1900) & 87081 (4353) & 78617 (4472) \\
\hline Data completeness (\%) & $100(100)$ & $97.9(95.1)$ & 100 (99.9) \\
\hline Average redundancy & $39.5(40.5)$ & $7.0(6.0)$ & $18.5(18.0)$ \\
\hline Mosaicity $\left({ }^{\circ}\right)$ & 0.08 & 0.09 & 0.05 \\
\hline
\end{tabular}




\begin{tabular}{|c|c|c|c|}
\hline Average $\| \sigma(I)$ & $41.0(7.4)$ & $12.9(1.7)$ & $14.0(3.0)$ \\
\hline Solvent content (\%) & 47 & 42 & 57 \\
\hline Matthews coefficient ( $\left.\AA^{3} / \mathrm{Da}\right)$ & 2.32 & 2.13 & 2.32 \\
\hline $\boldsymbol{R}_{\text {merge }}^{\dagger}$ & $0.061(0.637)$ & $0.085(0.894)$ & $0.153(0.976)$ \\
\hline $\boldsymbol{R}_{\text {pim }}$ & $0.010(0.102)$ & $0.053(0.618)$ & $0.052(0.337)$ \\
\hline $\mathrm{CC} 1 / 2$ & $1.000(0.975)$ & $0.999(0.671)$ & $0.998(0.890)$ \\
\hline \multicolumn{4}{|c|}{ Structure refinement parameters } \\
\hline$R_{\text {work }}$ & 0.167 & 0.157 & 0.166 \\
\hline$R_{\text {free }}$ & 0.202 & 0.207 & 0.201 \\
\hline $\boldsymbol{R}_{\text {all }}$ & 0.168 & 0.157 & 0.166 \\
\hline Average $B$-factor $\left(\AA^{2}\right)$ & 33 & 32 & 26 \\
\hline RMSD bond lengths from ideal $(\AA)$ & 0.016 & 0.018 & 0.019 \\
\hline RMSD bond angles from ideal $\left({ }^{\circ}\right)$ & 1.7 & 1.8 & 1.8 \\
\hline Number of non-hydrogen atoms & 2481 & 8344 & 7030 \\
\hline $\begin{array}{l}\text { Number of dimers per asymmetric } \\
\text { unit (chains) }\end{array}$ & $1 \mathrm{NKR}-\mathrm{P} 1(\mathrm{AB})$ & 4 NKR-P1 (AB, CD, EF, GH) & $\begin{array}{c}1 \text { LLT1 (AB), } 2 \text { NKR-P1 (CD, } \\
\text { EF) }\end{array}$ \\
\hline $\begin{array}{l}\text { Number of water molecules in the } \\
\text { asymmetric unit }\end{array}$ & 225 & 682 & 691 \\
\hline $\begin{array}{l}\text { Positions of modeled GIcNAc } \\
\text { residues }\end{array}$ & AB 116, AB 169 & $\begin{array}{l}\text { C 116, ABCEFGH 157, } \\
\text { ABCDEFGH } 169\end{array}$ & $\begin{array}{c}\text { AB 95, AB 147, } \\
\text { F 116, CDF 157, CDEF } 169\end{array}$ \\
\hline $\begin{array}{l}\text { Ramachandran statistics: residues } \\
\text { in favored regions (\%); number of } \\
\text { outliers }{ }^{48}\end{array}$ & $98 ; 0$ & $98 ; 0$ & $98 ; 0$ \\
\hline
\end{tabular}

${ }^{\dagger} R_{\text {merge }}=\sum_{\mathrm{h}} \sum_{\mathrm{i}}\left|I_{\mathrm{hi}}-\left\langle I_{\mathrm{h}}\right\rangle\right| / \sum_{\mathrm{h}} \sum_{\mathrm{i}} I_{\mathrm{hi}}, \quad R_{\mathrm{p} . \mathrm{i} . \mathrm{m} .}=\sum_{\mathrm{h}} \sum_{\mathrm{i}}\left(n_{\mathrm{h}}-1\right)^{-1 / 2}\left|I_{\mathrm{hi}}-\left\langle I_{\mathrm{h}}\right\rangle\right| / \sum_{\mathrm{h}} \sum_{\mathrm{i}} I_{\mathrm{hi}}, \quad$ and
$R=\sum_{\mathrm{h}}|| F_{\mathrm{h}, \text { obs }}|-| F_{\mathrm{h}, \text { calc }}|| / \sum_{\mathrm{h}}\left|F_{\mathrm{h}, \mathrm{obs}}\right|$, where $I_{\mathrm{hi}}$ is the observed intensity, $\left\langle I_{\mathrm{h}}\right\rangle$ is the mean intensity of multiple observations of symmetry-related reflections, while $F_{\mathrm{h}, \mathrm{obs}}$ and $F_{\mathrm{h} \text {,calc }}$ are the observed and calculated structure factor amplitudes, respectively. $R_{\text {work }}$ is the $R$ factor calculated on $95 \%$ of reflections excluding a random subset of $5 \%$ of reflections marked as "free". The final structure refinement was performed on all observed structure factors.

\section{Human NKR-P1 homodimer is similar to murine dectin-1 homodimer}

The asymmetric unit of NKR-P1_glyco contains two monomers, whereas the asymmetric unit of NKR-P1_deglyco contains eight NKR-P1 monomers. All these monomers are arranged into very similar homodimers, with pairwise RMSD on $\mathrm{C}^{\alpha}$ atoms up to $0.5 \AA$ (Fig. 2b). However, these homodimers have an unexpected configuration: they do not follow the usual dimerization mode observed for CTLDs of the CLEC2 ligands such as CD69 or LLT1 with helix $\alpha 2$ at the dimerization interface. Instead, the dimerization interface of NKR-P1 is formed by helix $\alpha 1$, as in the murine C-type lectin-like pattern recognition receptor dectin- $1^{49}$, 
with which human NKR-P1 shares only 32\% sequence identity of the CTLD (Fig. 2c). The RMSD of the $C^{\alpha}$ atoms between NKR-P1 and dectin-1 dimers is $3.7 \AA$ in the overlapping region (matching 196 from the total of 250 residues of the NKR-P1 dimer). The structurally distinct region mainly covers helices $\alpha 2$, whose positions differ up to $7 \AA$ between NKR-P1 and dectin-1. We also observed a highly similar arrangement with helix a1-centered dimerization interface in the structure of a covalent disulfide dimer of rat NKR-P1B receptor ectodomain $^{50}$ (Fig. S1a) with $1.4 \AA$ RMSD of the $\mathrm{C}^{\alpha}$ atoms between these two dimers (PDB ID 5J2S, manuscript in preparation). On the contrary, the non-classical dimer of mouse NKRP1B observed in the PDB ID 6E7D structure ${ }^{42}$ has an entirely different overall arrangement (Fig. S2a).

The dimerization interface of the NKR-P1 homodimer consists of six protein-protein and several water-mediated hydrogen bonds (Tab. S1), a peptide bond interaction via delocalized electrons (Lys126-Glu127), and a small hydrophobic core comprising Leu119, Ala120, and lle168 from both chains (Fig. S3). The contact surface area is ca. $500 \AA^{2}$. Compared with the helix a2-centered LLT1 dimer (7-12 hydrogen bonds, stronger hydrophobic core, 500-800 $\AA^{2}$ contact surface area) $)^{43}$, the helix a1-centered NKR-P1 dimer forms through a smaller contact surface area with fewer contact residues.

a

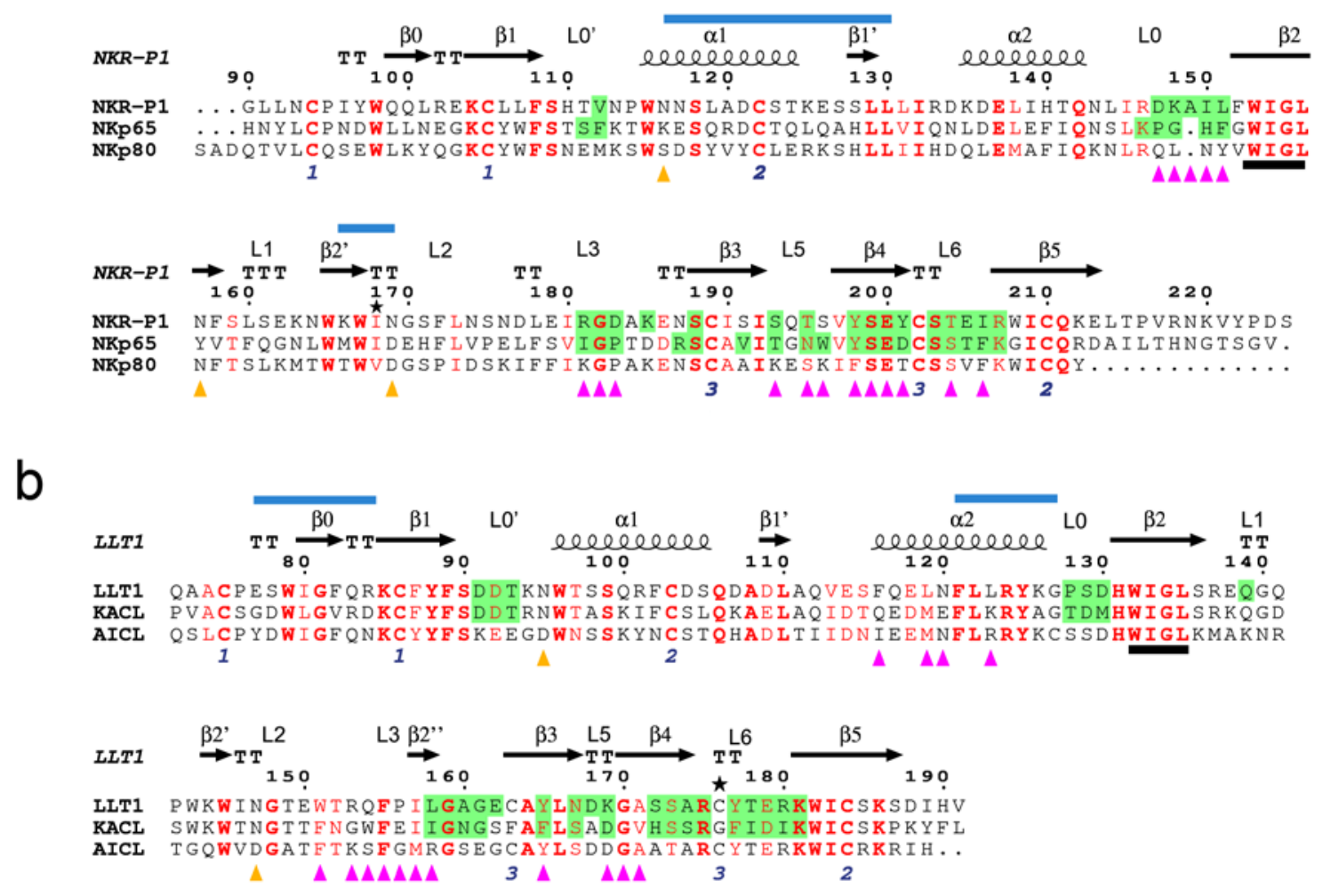

Figure 1. Sequence alignment of human NKC-encoded receptor:ligand pairs showing shared structural and functional sequence motifs. Secondary structure elements and loop regions (L) are denoted for NKR-P1 and LLT1 above the alignments. The paired numbers at the bottom indicate the disulfide pairs in the NKR-P1 and LLT1 structures; asterisks mark His176Cys mutation in LLT1 and Ile168 residue in NKR-P1. The predicted N-glycosylation sites of NKR-P1 and LLT1 are denoted with orange triangles. The conserved WIGL motifs are underlined in black. Blue lines above the sequence indicate the regions 
bioRxiv preprint doi: https://doi.org/10.1101/2021.06.16.448687; this version posted June 16, 2021. The copyright holder for this preprint (which was not certified by peer review) is the author/funder. All rights reserved. No reuse allowed without permission.

involved in forming the non-covalent dimers of NKR-P1 or LLT1. Conserved residues are marked red; bold letters denote strictly conserved residues. (a) Sequence alignment of CTLDs of human NKR-P1-related NK cell receptors, i.e., human NKR-P1, NKp65, and NKp80. NKR-P1 residues contacting LLT1 in the NKR-P1:LLT1 complex in the primary binding mode, and NKp65 residues contacting KACL in the NKp65:KACL complex, are highlighted in green. Purple triangles indicate NKR-P1 residues that engage LLT1 in the NKR-P1:LLT1 complex in the secondary binding mode. (b) Sequence alignment of CTLDs of LLT1related human CLEC2 ligands, i.e., LLT1, KACL, and AICL. LLT1 residues contacting NKR-P1 in the NKR-P1:LLT1 complex in the primary binding mode, and KACL residues contacting NKp65 in the NKp65:KACL complex, are highlighted in green. Purple triangles indicate LLT1 residues that engage NKR-P1 in the NKR-P1:LLT1 complex in the secondary binding mode.
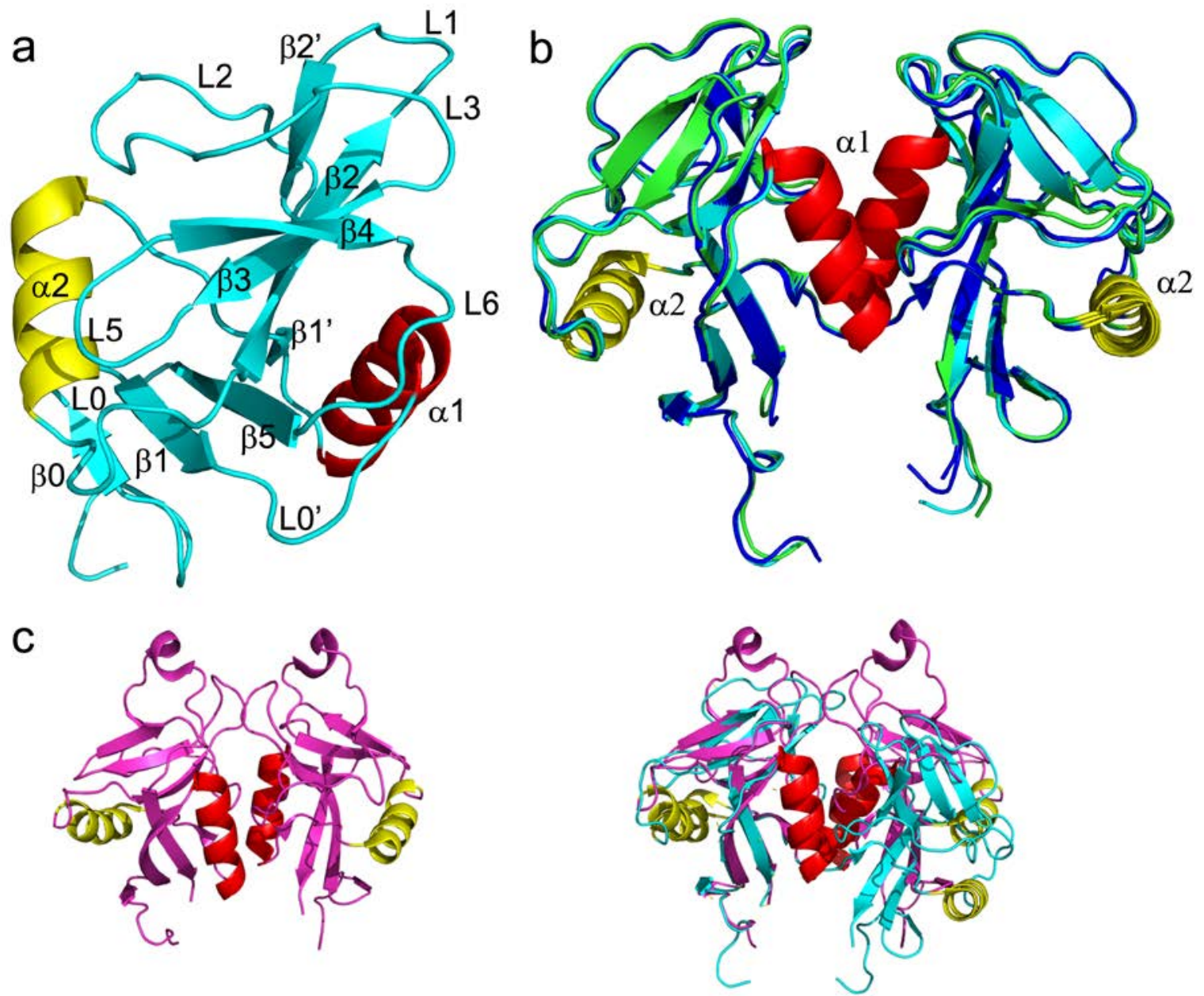

dectin-1

helix $\alpha 1$-centered dimer

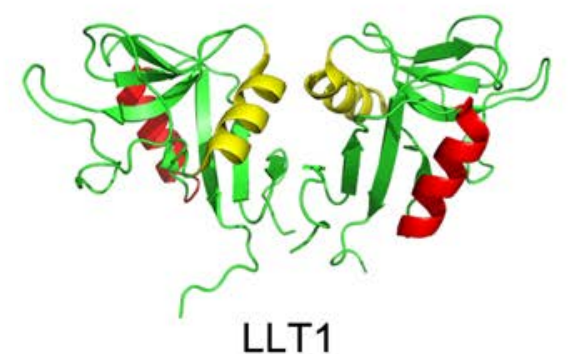

helix $\alpha 2$-centered dimer

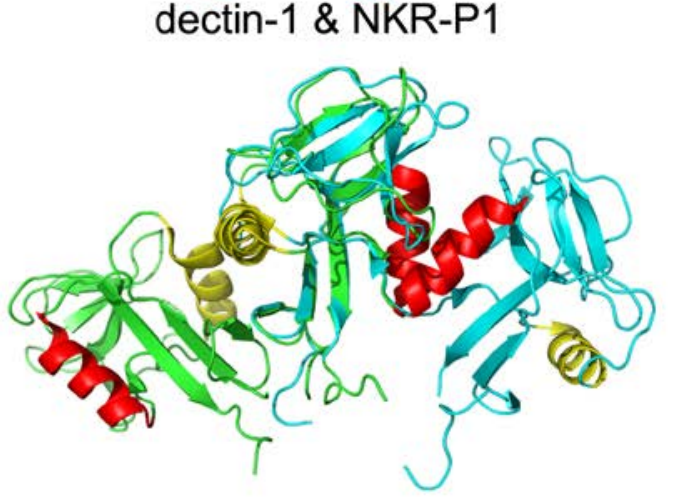

LLT1 \& NKR-P1

Figure 2. The structure of human NKR-P1 shows a unique dimerization interface. (a) Ribbon diagram of the NKR-P1 CTLD. Secondary structure elements are labeled in different colors: helix $\alpha 1$ is red, helix $\alpha 2$ is yellow, and $\beta$-strands and loops are cyan. (b) Comparison between NKR-P1 dimers formed by the glycosylated (cyan), deglycosylated free (green), and LLT1bound (blue) forms of NKR-P1. (c) Comparison between helices a1- and a2-centered dimerization of murine dectin-1 (PDB ID 2CL8, magenta) and human LLT1 (PDB ID 4QKI, green), respectively; helices $\alpha 1$ and $\alpha 2$ are shown in red and yellow. Structural alignments of dectin-1 and NKR-P1 homodimers and LLT1 and NKR-P1 homodimers, prepared by aligning only one monomer from each dimer, are shown on the right-hand side. Although the CTLD fold is conserved in each pair of the aligned monomers, the helix $\alpha 1$ - and helix $\alpha 2$-centered dimers show inverse arrangement. 
Glycosylation of human NKR-P1 affects its dimerization

The NKR-P1 ectodomain contains three potential N-glycosylation sites at residues Asn116, Asn157, and Asn169 (Fig. 1a). Glycosylation at Asn169 is visible in the electron density maps of both NKR-P1_glyco and _deglyco structures: in NKR-P1_glyco, the complete GlcNAC $_{2} \mathrm{Man}_{5}$ carbohydrate chain is localized in chain $A$, whereas a partial $\mathrm{GICNAC}_{2} \mathrm{Man}_{3}$ chain is localized in chain B (Fig. S3a). In NKR-P1_deglyco, a single GlcNAc unit remaining at Asn169 can be well-identified in all eight NKR-P1 chains. Interestingly, the localized first GlcNAc units linked at Asn116 in NKR-P1_glyco and the overlapping GlcNAc units at Asn116 and Asn157 remaining in NKR-P1_deglyco participate in dimerization contacts with residues of helices $\alpha 1$ and regions $\beta 2, L 1$, and $\beta 2$ ' of the opposite subunit of the NKR-P1 homodimer (Fig. S3b). In NKR-P1_glyco, five hydrogen bonds between Asn116:GlcNAc and the opposite subunit stabilize the helix a1-centered homodimer (Tab. S1). The contact surface area between the opposite chain of NKR-P1 and the localized GICNAc unit is approximately $125 \AA^{2}$

In NKR-P1_deglyco, density at the homodimer interface can accommodate the remaining GICNAc unit of either Asn116 of one chain or Asn157 of the opposing chain of the NKR-P1 dimer (for details, see Methods, Tab. 1, and Fig. S3c). Conversely, in NKR-P1_glyco, the first GlcNAc unit at Asn116 is well defined in electron density in both chains, $A$ and $B$, of the NKR-P1 dimer, while no electron density was found for glycosylation at Asn157. This suggests that although the glycosylation on either Asn116 or Asn157 can contribute to the dimer formation, dimerization might be impaired by steric hindrance if both $\mathrm{N}$-glycans are present simultaneously. To test this hypothesis, we expressed NKR-P1 S159A mutant, thus abrogating glycosylation on Asn157. The overall fold of the mutant is comparable to the wildtype NKR-P1, as assessed by CD spectroscopy (Fig. S4a). Indeed, when analyzed by analytical ultracentrifugation, the mutant protein exhibited substantial levels of oligomeric species (Fig. S4b), whereas the wild-type NKR-P1 ectodomain is purely monomeric ${ }^{51}$. Thus, glycosylation heterogeneity may affect the propensity of human NKR-P1 to dimerize and, therefore, its ability to form multimeric complexes with LLT1.

\section{Structure of the human NKR-P1:LLT1 complex}

The crystal structure of the NKR-P1:LLT1 complex is formed by deglycosylated NKR-P1 and LLT1 ectodomains. The asymmetric unit of the crystal contains a complex of dimeric NKRP1 with dimeric LLT1 and an extra dimer of NKR-P1 (Fig. 3a). These NKR-P1 dimers have the same helix a1-centered dimerization interface as the structures of the unbound NKR-P1 dimers described above (Fig. 2b). The LLT1 dimer retains the expected helix a2-centered dimerization mode (Fig. 2c), identical to that described previously in unbound LLT1 
structures $^{43}$. LLT1 has clearly identifiable GIcNAc units at residues Asn95 and Asn147. The NKR-P1 glycosylation observed in the electron density of the complex matches the glycosylation identified in the NKR-P1_deglyco structure.

The LLT1 homodimer engages its partner bivalently, i.e., one dimer interacts with two NKRP1 dimers related by crystallographic symmetry: each LLT1 monomer binds to a different subunit of a distinct NKR-P1 homodimer (Fig. 3a). There is no apparent induced fit of the binding partners - the RMSD of $C^{\alpha}$ atoms between the non-interacting and the interacting NKR-P1 dimers (NKR-P1_glyco and the complex) is $0.5 \AA$, and that of LLT1 (PDB ID 4QKH and the current complex) is $0.7 \AA$. Moreover, the $\mathrm{N}$-linked glycosylation chains do not directly contribute to the interaction.

\section{LLT1 engages NKR-P1 in two distinct interaction modes}

NKR-P1 and LLT1 establish two types of contact in this structure - the primary (LLT1 chain B:NKR-P1 chain D) and the secondary (LLT1 chain A:NKR-P1 symmetry-related chain C) interaction modes. The primary interaction mode matches well the structure of the homologous human NKp65:KACL complex (PDB ID 4IOP) ${ }^{37}$ - the RMSD of $C^{\alpha}$ atoms of the two complexes is $1.3 \AA$ (one chain of the receptor and one chain of the ligand in each case, Fig. 3b, lower left). Similarly, in the structure of mouse NKR-P1B complexed with MCMV immunoevasin $\mathrm{m} 12$, the observed interaction interface matches the primary mode in the present structure of the human NKR-P1 complex, although the area covered by the m12 protein is considerably larger (Fig. S1b,c) ${ }^{41}$. The recently described structure of the mouse NKR-P1B:Clrb complex (PDB ID 6E7D) ${ }^{42}$ also showed an interaction interface common for both NKR-P1 receptors (Fig. S2b,c). However, the receptor:ligand arrangement observed in the second interaction mode of the NKR-P1:LLT1 complex is unique and differs from all known homologous complexes in the orientation of the ligand (Fig. 3b, lower right, Fig. S2b). The interaction interfaces of human NKR-P1 involved in both primary and secondary interaction modes are very similar. They are formed mainly by the membrane-distal residues of the L0, L3, L5, and L6 loops and by $\beta 3$ and $\beta 4$ strands (Fig. 3c,d) that form a flat surface for interaction with LLT1. By contrast, in LLT1, the primary and secondary interaction interfaces are substantially different, albeit sharing a small number of residues. While the loops L0', L0, L3, L5, L6 and strands $\beta 3$ and $\beta 4$ form the primary interaction patch of LLT1 (Fig. 3c), residues of the loops $L 2$ and $L 5$, strand $\beta 2$ " and helix $\alpha 2$ are involved in the second interaction interface (Fig. 3d). Both interaction modes place the membrane-proximal parts of the receptor and the ligand on opposite sides of the complex, creating a plausible model for interaction between two neighboring cells.

The primary interaction mode is established through nine direct and several water-mediated hydrogen bonds, in addition to two charge-supported and $\pi-\pi$ stacking (Tyr201-Arg175) 
interactions, with a total contact surface area of ca. $800 \AA^{2}$ (Tab. S1). The four strongest bonds occur between the NKR-P1 residues Arg181, Tyr201, Lys148, and Ser199 and the LLT1 residues Glu179, Glu162, Ser129, and Tyr177, respectively (Fig. 3c). The second interaction mode is established through five direct hydrogen bonds, two charge-supported and a hydrophobic (LLT1:Pro156 - NKR-P1:Ala149, Leu151) interaction, with a total contact surface area of ca. $550 \AA^{2}$ (Tab. S1). The three strongest bonds occur between the NKR-P1 residues Asp147, Ser199, and Arg181 and the LLT1 residues Arg153, Lys169, and Asn120, respectively (Fig. 3d).

Besides interaction with LLT1, NKR-P1 dimers bound in primary and secondary mode also have non-negligible mutual accessory contact (NKR-P1 chain D and NKR-P1 chain C in symmetry-related position). The interface area has $440 \AA^{2}$ and is established through seven direct hydrogen bonds (Tab. S1). Four residues are towering above the interface: Asn143 and Arg146 of chain D and Asn174 and Asn176 of chain Csym (none of the asparagine residues is a glycosylation site). The interface also has several water-mediated contacts. 
bioRxiv preprint doi: https://doi.org/10.1101/2021.06.16.448687; this version posted June 16, 2021. The copyright holder for this preprint (which was not certified by peer review) is the author/funder. All rights reserved. No reuse allowed without permission.

a

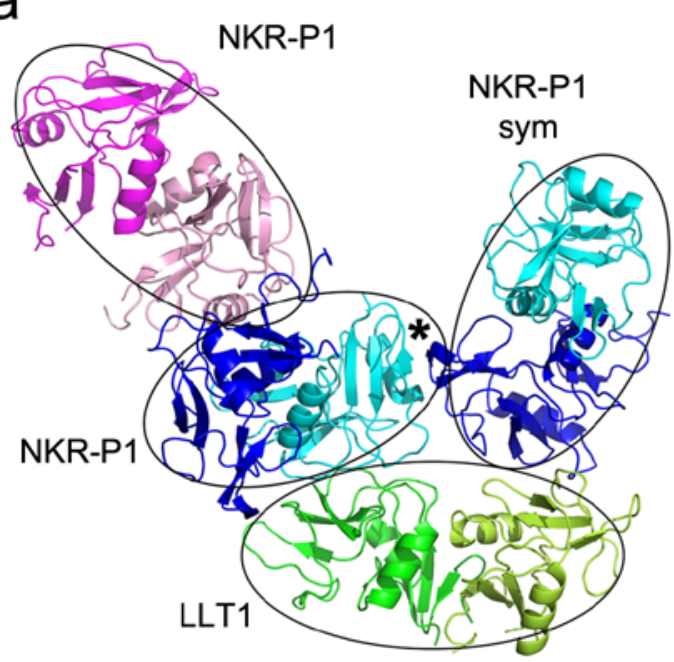

C Primary mode

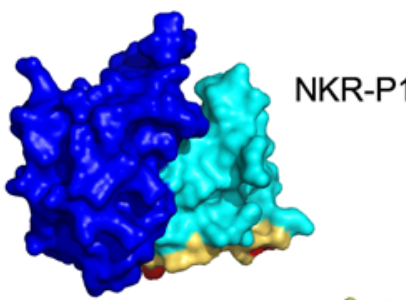

LLT1

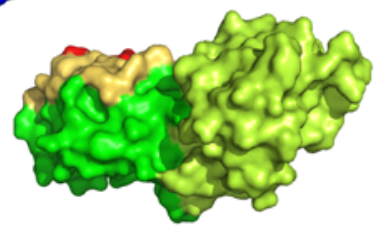

d Secondary mode

NKR-P1
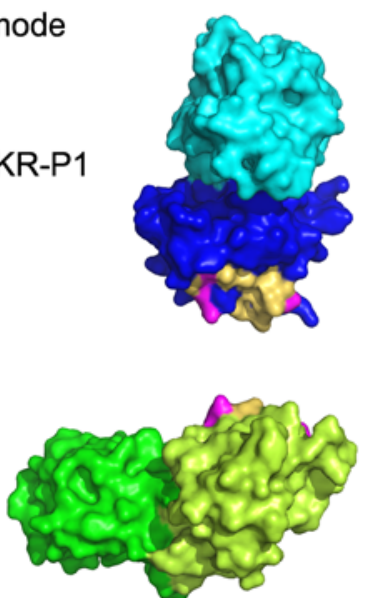

LLT1 b
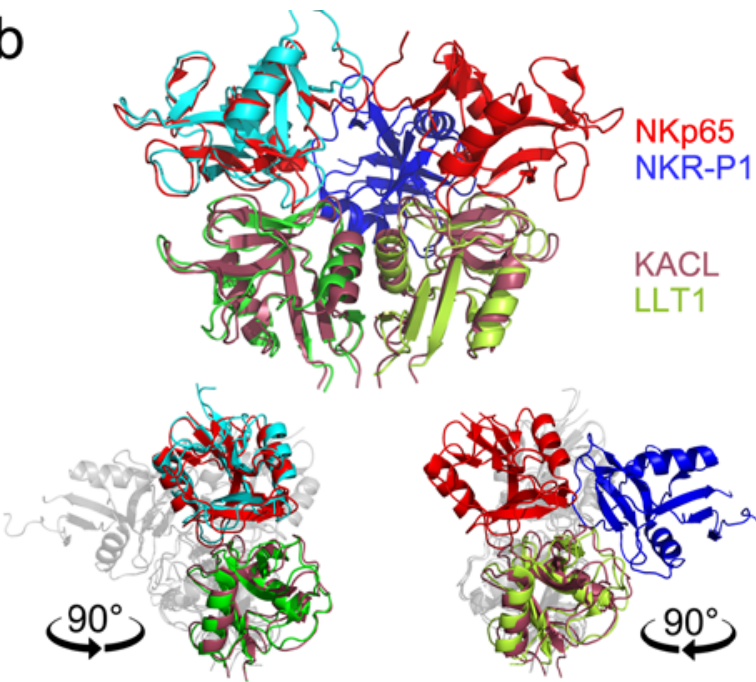

Primary mode

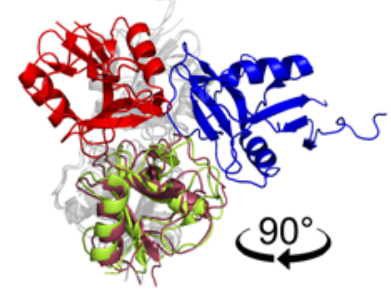

Secondary mode
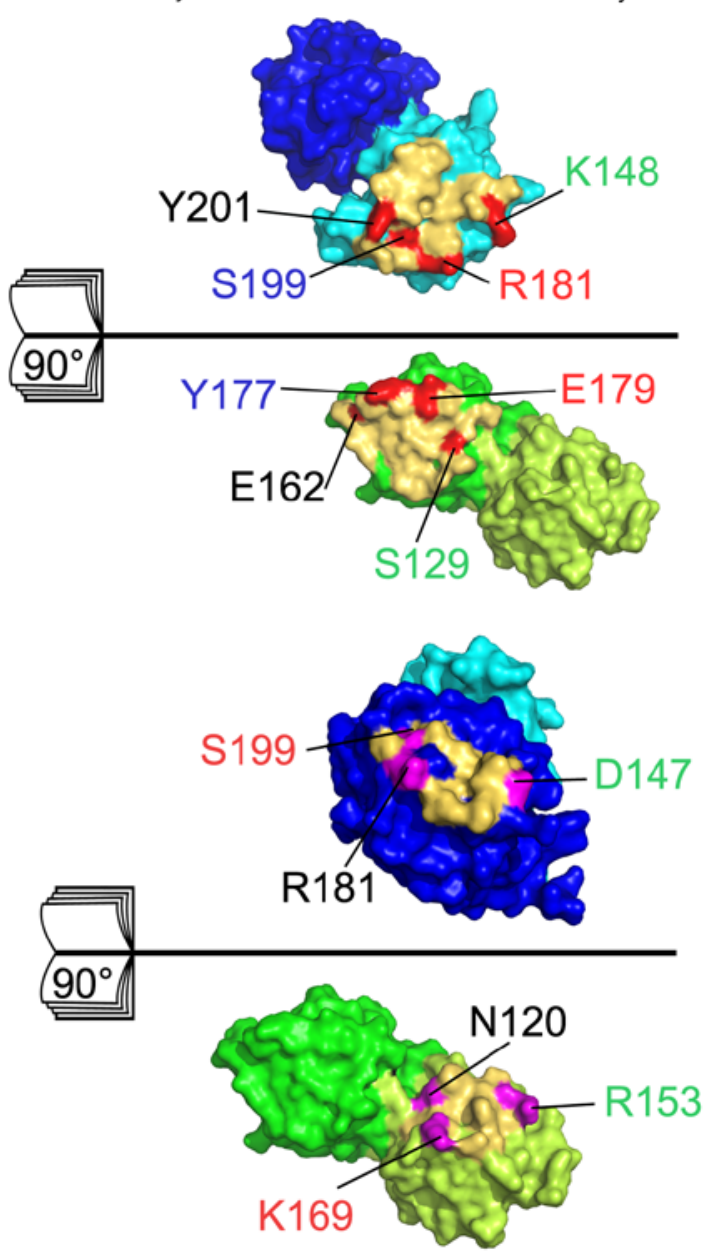

Figure 3. The structure of the NKR-P1:LLT1 complex shows two distinct binding modes. (a) The overall organization of the complex crystal structure. The LLT1 dimer (green/lemon) contacts the NKR-P1 dimer, formed by the blue monomer and the cyan monomer. The second blue-cyan NKR-P1 dimer is related to the first by crystal symmetry. The cyan NKR-P1 monomer interacts with LLT1 in the primary interaction mode, whereas the blue NKR-P1 monomer engages LLT1 using the secondary interaction interface. Black asterisk marks the mutual accessory contact of NKR-P1 bound in primary and secondary mode. Additionally, the asymmetric unit of the crystal contains another NKR-P1 dimer (pink/magenta) lacking contact with LLT1. (b) Overall comparison of the structure of dimeric KACL (purple) in complex with two NKp65 monomers (red; PDB ID 4IOP) and the structure of the LLT1 dimer (green/lemon) with the two interacting NKR-P1 molecules in the primary (cyan, left side) and secondary (blue, right side) binding modes. Comparison with only the primary or secondary NKR-P1:LLT1 interaction modes is highlighted in the lower section (both in a side view, using $90^{\circ} \mathrm{y}$-axis rotation). (c) NKR-P1:LLT1 primary interaction interface. Contact residues within $5 \AA$ distance are colored in yellow. Amino acids forming the four strongest contacts are highlighted in red. (d) NKR-P1:LLT1 secondary interaction interface. Contact residues within $5 \AA$ distance are colored in yellow. Amino acids forming the three strongest contacts are highlighted in magenta. 


\section{NKR-P1:LLT1 complex formation in solution}

To characterize the size and shape of the NKR-P1:LLT1 complex in solution, we have performed analytical ultracentrifugation (AUC), microscale thermophoresis (MST), and smallangle X-ray scattering coupled to size-exclusion chromatography (SEC-SAXS) experiments. The acquired AUC data are concentration-dependent and reflect the dynamic nature of this interacting system, similar to the results from the SEC-SAXS measurements. The free human NKR-P1 ectodomain is monomeric, with a sedimentation coefficient $s_{20, w}$ of $2.1 \mathrm{~S}$ corresponding to an estimated molecular mass of $18 \mathrm{kDa}$, matching the expected value of 17.5 kDa closely. The LLT1 ectodomain forms a stable non-covalent dimer (2.9 S) that does not dissociate into monomers, except in very low concentration (previously characterized in Skálová et al., 2015 43 and Bláha et al., 201751). When increasing the loading concentration of the NKR-P1:LLT1 equimolar mixture, the sedimentation coefficient of the complex increases as well, reaching an $\mathrm{S}_{20, w}$ value of $3.7 \mathrm{~S}$ at the highest analyzed concentration (Fig. S4c). This value, when corrected for non-ideality caused by the high protein loading concentration used $(18 \mathrm{mg} / \mathrm{ml})$, corresponds to an estimated zero-protein-concentration sedimentation coefficient $\mathrm{S}_{20, w}$ value of $4.5 \mathrm{~S}$, and a moderately elongated particle with approximate dimensions of $10-15 \times 4-5 \times 4-5 \mathrm{~nm}$. That compares well with the 8-10 8 $6 \times 4-5 \mathrm{~nm}$ dimensions expected for the possible NKR-P1:LLT1 interaction assemblies observed in the complex's crystal structure, i.e., monomer:dimer:monomer or dimer:dimer (note that while $\mathrm{N}$-glycan chains are not present in the deglycosylated complex's structure, they were present during all our analyses in solution).

\section{Secondary interaction mode is involved in NKR-P1:LLT1 binding in solution}

To understand whether the secondary interaction mode observed within the crystal structure is also utilized in the solution, we designed an N120R, R153E, K169A triple mutant of LLT1 (LLT1 ${ }^{\mathrm{SIM}}$ ), thus abolishing the three strongest contacts in the LLT1 secondary interaction interface (Fig. 3d). The LLT1 ${ }^{\text {SIM }}$ mutant was expressed and purified in the same way as the wild-type LLT1, and it also displayed a comparable CD spectrum (Fig. S4a). NKR-P1:LLT1 ${ }^{\text {SIM }}$ equimolar mixture concentration series reached an $\mathrm{s}_{20, w}$ value of $3.3 \mathrm{~S}$ (corresponding to an estimated $\mathrm{s}^{0}{ }_{20, w}$ value of $3.9 \mathrm{~S}$ ), clearly showing the formation of the complex of a smaller size compared to the wild-type NKR-P1:LLT1 mixture, possibly a monomer:dimer assembly (Fig. S4d). By integrating the whole continuous size distribution c(s) curves and plotting the resulting weight-average $S$ values against the LLT1 proteins' concentrations used, binding isotherms were constructed for both dilution series and firstly best-fit to the simplest heteroassociation binding model $A+B \Leftrightarrow A B$, where $A$ is the LLT1 dimer and $B$ is the NKR-P1 monomer (Fig. S5a). LLT1 ${ }^{\text {SIM }}$ showed about three-fold weaker overall affinity than the wild- 
type LLT1, which was further corroborated by independent MST analysis using fluorescently labeled NKR-P1 titrated with LLT1 or LLT1 ${ }^{\mathrm{SIM}}$ (Fig. S5b). To analyze the difference between wild-type LLT1 and LLT1 ${ }^{\text {SIM }}$ concerning the binding of the second NKR-P1 monomer, we also best-fit the data using the $A+B \Leftrightarrow A B+B \Leftrightarrow B A B$ model (Fig. S5c). While for the wild-type LLT1 this improved the fit and provided two different $K_{D}$ values, possibly corresponding to primary and secondary interaction modes, for LLT1 ${ }^{S I M}$, the fitted $K_{D}$ values remained unchanged, disproving binding of the second NKR-P1 monomer. Similarly, when the AUC and MST data were globally fit together with the AUC parameters fixed at the previously best-fit values, the LLT1 ${ }^{\text {SIM }}$ MST data could be fitted equally well with both $A B$ and BAB model while the fit is poor for the AB model in the case of wild-type LLT1 (Fig. S5d). Taken together, our data show that the secondary interaction interface is not just a crystal contact, but it contributes significantly to the overall affinity of the NKR-P1:LLT1 interaction.

\section{SEC-SAXS analysis confirms NKR-P1:LLT1 higher-order complex formation}

The observation of assemblies of even higher-order is unlikely in the AUC sedimentation velocity experiment, given the fast kinetics of this interaction, the long time scale of the experiment, and the fact that protein concentration steadily decreases along the sedimentation boundary. However, the NKR-P1:LLT1 complex's crystal structure suggests a chain-like arrangement of receptor:ligand dimers. To determine if any such assemblies exist in the solution, we performed SEC-SAXS analysis of the NKR-P1:LLT1 equimolar mixture at $15 \mathrm{mg} / \mathrm{ml}$ loading concentration. Two distinct peaks of absorbance at $280 \mathrm{~nm}$, corresponding to two distinct SAXS peaks, were observed in the SEC-SAXS experiment (Fig. 4). For analysis and ab initio modeling of the SAXS envelopes, we sampled and merged four data intervals from the first peak and two data intervals from the second peak (Figs. 4 and S6). The radius of gyration calculated from the SAXS signal steadily decreases with the retention volume, with two small plateaus in the main peak, suggesting a dynamic balance between complex formation and dissociation (Fig. 4). As a result, the merged scattering curves could not be fitted well to scattering curves simulated from any single structure of the NKRP1:LLT1 complex or its components. For further analysis, we constructed models of assemblies of NKR-P1:LLT1 complex in varying lengths (number of protein chains), considering all possible permutations of the order of the molecules using only primary, only secondary, or both interaction modes in alternating fashion. The resultant library of all these models was then used by the OLIGOMER software to best-fit the observed SAXS experimental curves. By this approach, the sampled SAXS data fit reasonably well to the superimposed calculated scattering curves of groups of NKR-P1:LLT1 complex structures of higher stoichiometry $\left(x^{2} 1.29-3.47\right.$; note that complete glycosylation was not modeled but it contributed to the scattering; Fig. S6). Following the continuously decreasing radius of 
gyration calculated from the SAXS data, the stoichiometry of the best-fit structures of the NKR-P1:LLT1 complex continuously decreases with the retention volume as well. SAXS scattering curve of the first peak is best-fit with two to three LLT1 dimers interacting in a chain-like oligomer with two to three NKR-P1 dimers, the curve of the second peak to one LLT1 dimer interacting with one NKR-P1 dimer in primary or secondary mode. The most abundant components of the superimposed structures are best-fit to the averaged ab initio SAXS envelopes in Fig. S6. Notably, OLIGOMER generally favored assemblies utilizing the secondary interaction mode against models with the primary mode alone.

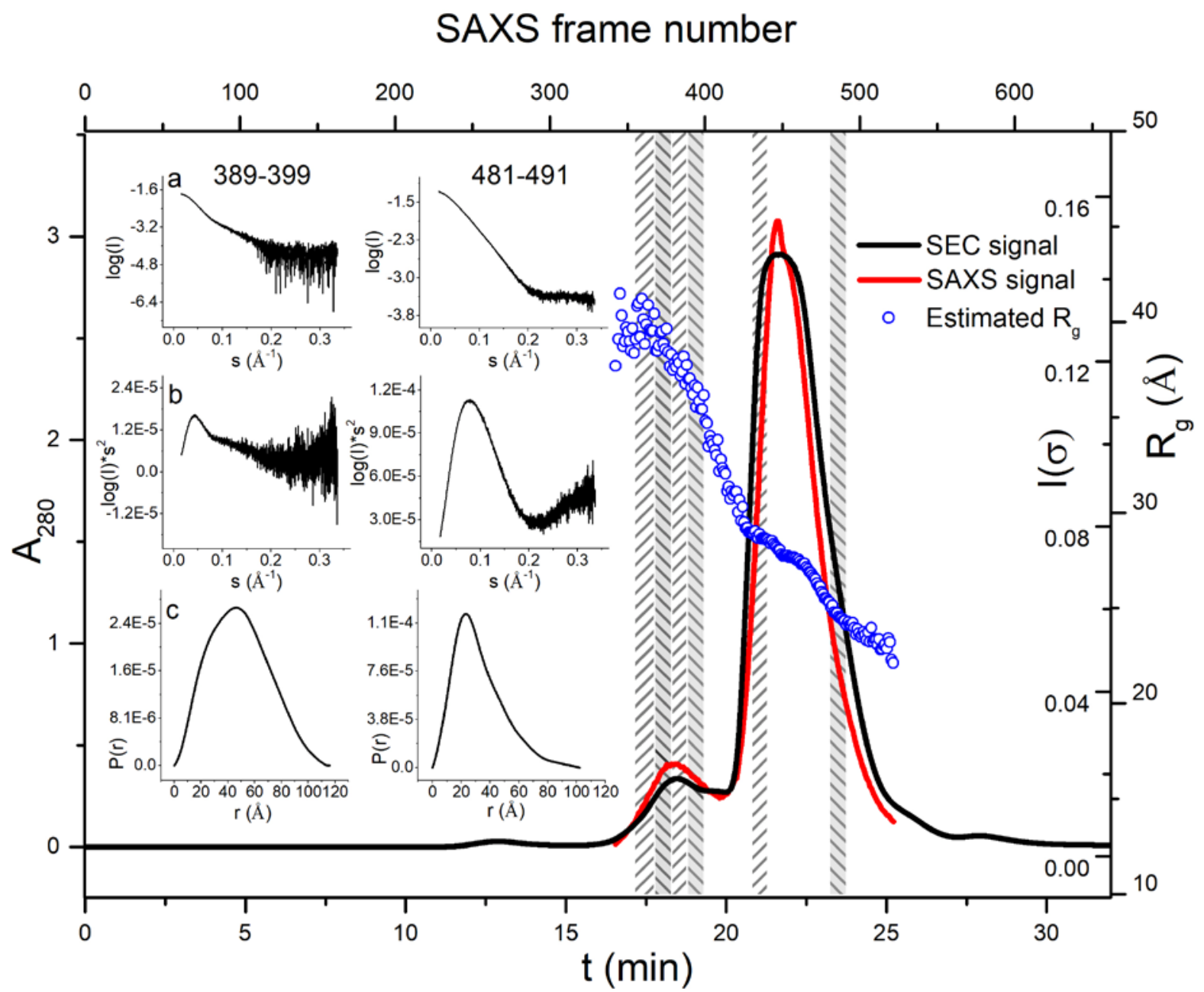

Figure 4. SEC-SAXS analysis of NKR-P1:LLT1 shows higher-order complex formation. Overlay of the size-exclusion chromatography profile (black line) and SAXS scattering signal (red line) for the NKR-P1:LLT1 equimolar mixture at $15 \mathrm{mg} / \mathrm{ml}$ loading concentration. Both signals show two distinct peaks. For each collected SAXS frame, the radius of gyration was calculated using AUTORG (blue circles). For the calculation of ab initio SAXS envelopes and further analysis by OLIGOMER (Fig. S6), six intervals of the SAXS data were selected and merged (frames 355-367, 368-378, 379-388, 389-399, 431-440, 481-491; denoted as columns with diagonal hatching). SAXS scattering curve (a), Kratky plot (b), and pair distance distribution function (c) for the intervals 389-399 and 481-491 are shown in the inset for data quality assessment of the merged data. 


\section{NKR-P1 signal transduction in live cells}

To answer the question of the biological relevance of these higher-order complexes in the context of a live cell, we performed single-molecule localization microscopy studies with NKR-P1-expressing cell line and quantified the surface distribution of NKR-P1 with respect to LLT1 or LLT1 ${ }^{\text {SIM }}$ binding. Next, we analyzed the effect of soluble LLT1 or LLT1 ${ }^{\text {SIM }}$ on the inhibitory potential of native NKR-P1 expressed on the surface of freshly isolated NK cells.

\section{LLT1 ligation induces NKR-P1 receptor clustering}

Full-length NKR-P1 transfectants generated using the piggyBac system were induced to express the receptor in a limited density to allow for single-molecule localization microscopy. Cells were then incubated in the presence or absence of soluble LLT1 or LL1 ${ }^{\text {SIM }}$, fixed, and labeled with anti-NKR-P1 Alexa-647 mAb. Direct stochastic optical reconstruction microscopy (dSTORM) images were acquired, and Voronoi tessellation cluster analysis was used to assess the effect of soluble LLT1 on the nanoscale organization of NKR-P1 on the cell surface (Fig. 5a). In the absence of LLT1, we detected clusters of events with an average area of $1870 \pm 777 \mathrm{~nm}^{2}$ (Fig. 5b) and an average diameter of $41 \pm 7 \mathrm{~nm}$ (Fig. 5c), mostly clusters with a diameter ranging from 10 to $40 \mathrm{~nm}$ (Fig. $5 \mathrm{~d}$ ), indicative of Alexa-647 doubly labeled NKR-P1 homodimers (ca. $6 \mathrm{~nm}+10 \mathrm{~nm}$ linking error per each mAb $\pm 15 \mathrm{~nm}$ of localization precision). The addition of soluble LLT1 significantly increased the cluster diameter (to $47 \pm 6 \mathrm{~nm} ; \mathrm{p}<0.0001$, Fig. 5c,d), the cluster area $\left(2713 \pm 1180 \mathrm{~nm}^{2} ; \mathrm{p}<\right.$ 0.0001 , Fig. 5b) and the number of events detected in the clusters (38.8 $\pm 12.3 ; p<0.0001$, Fig. 5e), whereas the density of events in clusters remained unchanged, as expected (Fig. 5f). Furthermore, the effect of LLT1 ${ }^{\text {SIM }}$ addition on NKR-P1 nanoscale organization was statistically indistinguishable from the negative control while being significantly different from the effect of LLT1 addition. Therefore, the secondary interaction interface in LLT1 is necessary for the formation of larger nanoscale NKR-P1 clusters upon interaction. No significant difference in the total density of events per inner cell surface (evaluated area) was found (Fig. 5g), pointing to stable expression levels of NKR-P1 throughout the measurement. Consequently, the size of NKR-P1 nanoclusters increases (beyond one homodimer unit) not because of differences in NKR-P1 expression levels but because LLT1 cross-links two or more NKR-P1 homodimers. 


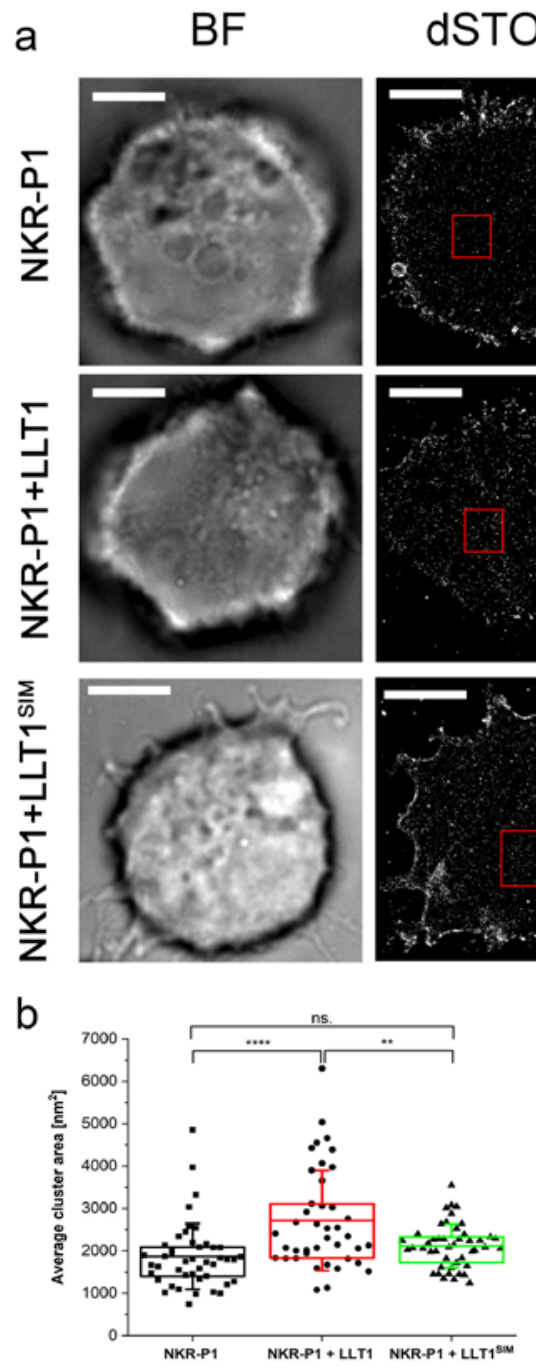

e

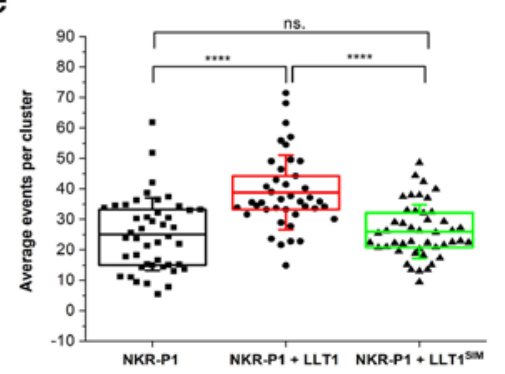

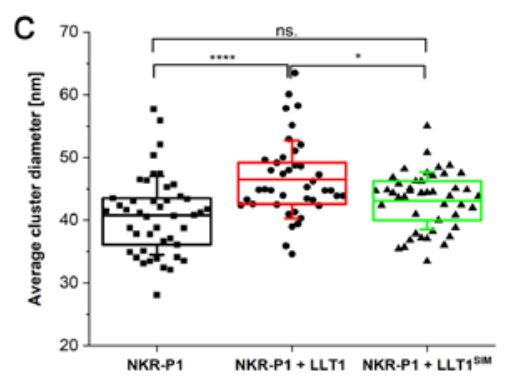

f

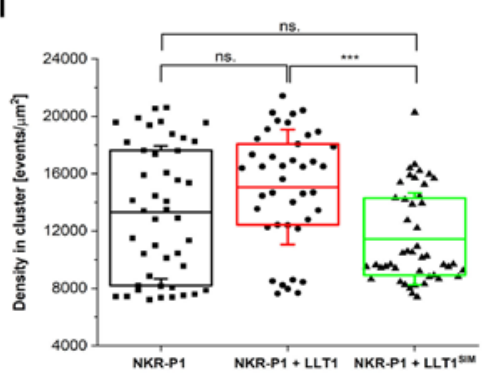

Cluster map
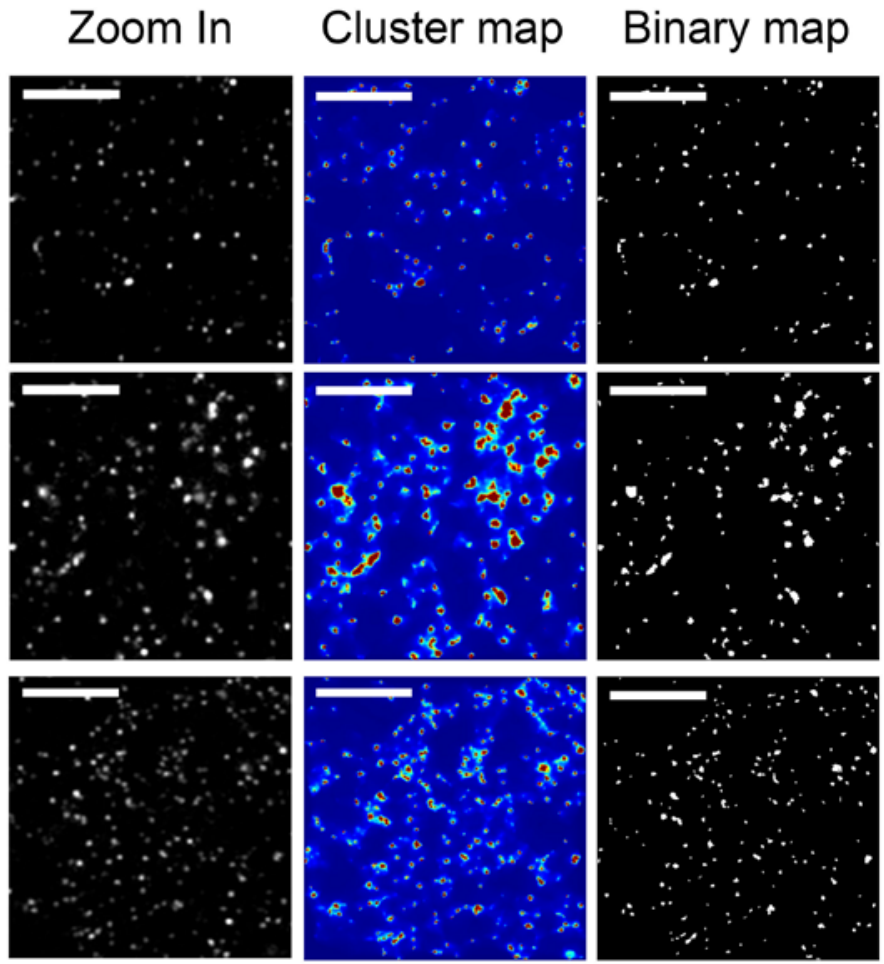

d

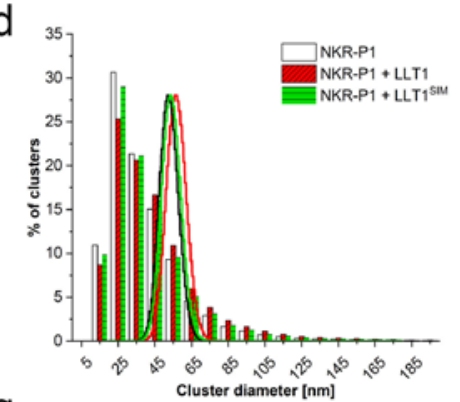

g

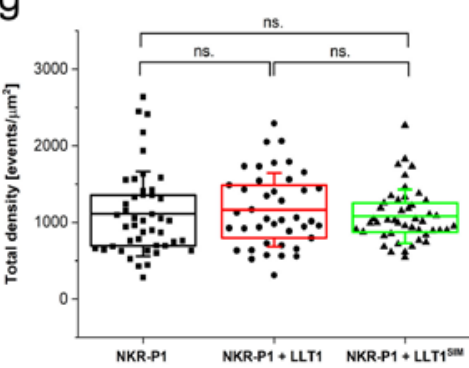

Figure 5. Soluble LLT1 affects NKR-P1 distribution on the cell surface. NKR-P1 stable transfectants were incubated in the presence or absence of soluble LLT1 or LLT1 ${ }^{\text {SIM }}$, and the cell surface distribution of NKR-P1 was monitored by super-resolution microscopy. (a) Representative brightfield (BF) and dSTORM images of full-length NKR-P1 HEK293 stable transfectants on PLL-coated slides incubated without (black) or with LLT1 (red) or LLT1 ${ }^{\text {SIM }}$ (green), fixed and stained with Alexa-647-labeled antiNKR-P1 mAb; scale bars represent $5 \mu \mathrm{m}$. The $10 \mu \mathrm{m}^{2}$ regions (red boxes in dSTORM images) are magnified and shown with corresponding cluster maps and binary maps, scale bars represent $1 \mu \mathrm{m}$. (b-g) Analysis of full-length NKR-P1 clustering: average cluster area (b), average cluster diameter (c), size distributions of cluster diameters overlaid with Poisson distribution functions (d), average events per cluster (e), density of events detected per cluster ( $f$ ) and total density of the detected events (g). In (b,c) and (e,f), each plotted point represents the mean value obtained from the analysis of the total inner surface of a single cell. The horizontal bar represents the overall mean value, the box represents the interquartile range, and the error bars

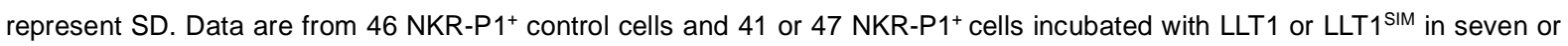
four independent experiments, respectively. One-way ANOVA with Bonferroni correction, ${ }^{\star} p<0.05,{ }^{* *} p<0.01,{ }^{* \star *} p<0.001$, $\star * \star * p<0.0001$, ns. non-significant. 


\section{LLT1 secondary interaction mode is required for NKR-P1 inhibitory signaling}

An NK cell-mediated cytotoxicity assay was performed to investigate further the influence of NKR-P1 cluster formation on cellular signalization. NK cells isolated from the blood of three different healthy donors were activated with IL-2 and mixed with the K562 target cells in a 40:1 effector:target cell ratio and incubated for 4 hours with PBS buffer as a negative control or with the soluble LLT1 or LLT1 ${ }^{\text {SIM }}$ (both in two different concentrations). In the absence of NKR-P1 ligand, K562 cells are well-susceptible to NK cell-mediated lysis (Fig. 6, PBS control, less than $10 \%$ live K562 cells). The lysis was substantially blocked in the presence of soluble LLT1, as expected. By contrast, the LLT1 ${ }^{\text {SIM }}$ variant with a mutated secondary interaction interface was incapable of blocking NK cell-mediated cytotoxicity. As observed by dSTORM on the cell surface (Fig. 5), LLT1 ${ }^{\text {SIM }}$ does not promote NKR-P1 cluster formation. This mutant also fails to signalize via the NKR-P1 inhibitory receptor pathway. Therefore, based on both the microscopy and the cytotoxicity assay data, we conclude that NKR-P1 clustering triggered by LLT1 ligation is biologically relevant and indispensable for cellular signalization.

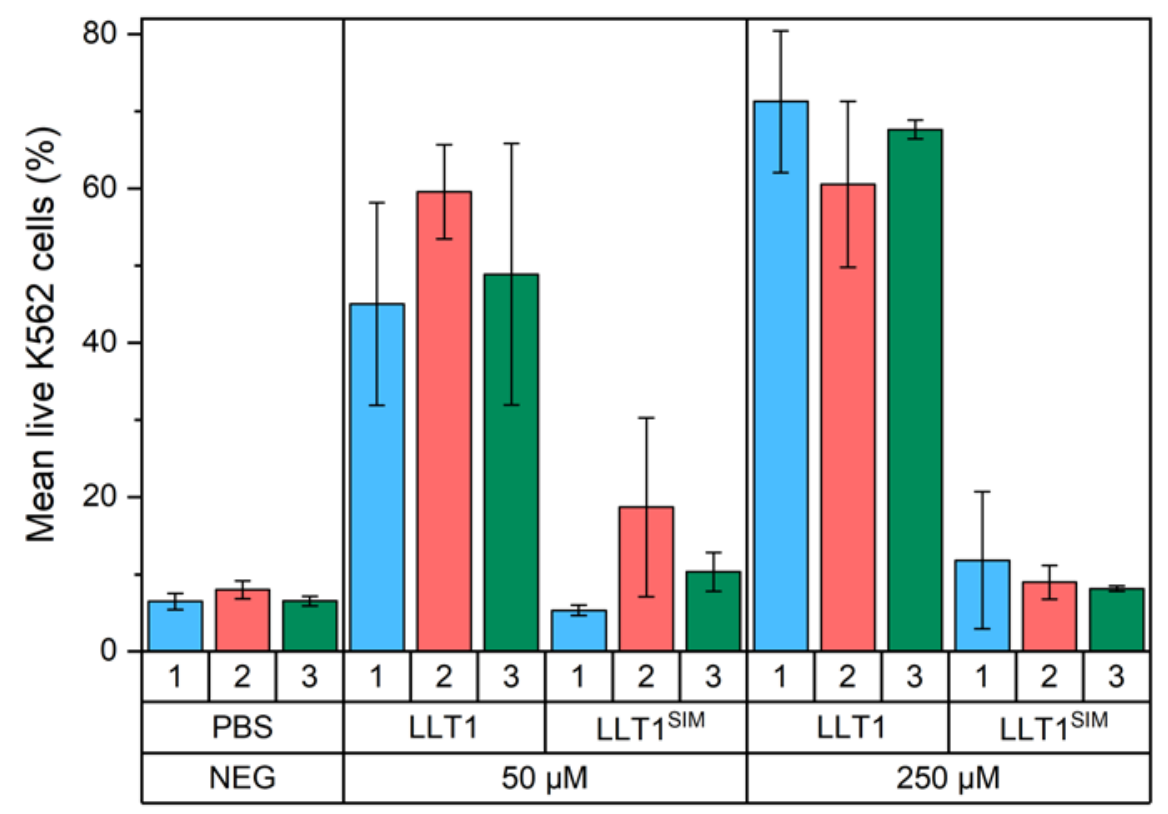

Figure 6. Inhibitory effect of soluble LLT1 on the NK cell-mediated cytotoxicity. NK cells from three different donors (blue, red, and green) and K562 target cells were incubated with PBS buffer only (negative control) or with the soluble LLT1 or LLT1 ${ }^{\text {SIM }}$ proteins, both in 50 and $250 \mu \mathrm{M}$ concentrations corresponding to the $1 \times$ and $5 \times K_{D}$ values, respectively, as analyzed by the AUC for the primary interaction mode (cf. Fig. S5). Results are represented as means of live K562 cells in each condition with standard deviation. When applicable, data were statistically evaluated by one-way ANOVA. Considering $p<0.05$ as statistically significant, the inhibitory effect of soluble LLT1 significantly differs from LLT1 ${ }^{\mathrm{SIM}}$, which does not differ from the condition without any NKR P1 ligand. 


\section{Discussion}

Comparison with homologous NK cell receptor:ligand complexes

Currently, there are only two similar CTL:CTL NK cell receptor:ligand complexes with known 3D structure: human NKp65:KACL ${ }^{37}$ and mouse NKR-P1B:Clrb ${ }^{42}$. These homologous complexes are topologically similar to the primary interaction mode of the NKR-P1:LLT1 complex (Fig. 3b). Sequence identity of the extracellular part of NKR-P1 with NKp65 is 33\% and with murine NKR-P1B 42\%, while sequence identity of the extracellular part of LLT1 with $\mathrm{KACL}$ is $49 \%$ and with murine Clrb $51 \%$. The structure of the human NKp65:KACL complex comprises two monomeric NKp65 units interacting separately and symmetrically with a dimeric KACL ligand. Crystal structure of the murine NKR-P1B:Clrb complex shows two Clrb dimers interacting with one NKR-P1B dimer placed between them (each Clrb dimer interacts with one of the two NKR-P1B chains). The arrangement of the murine complex is similar to the herein presented NKR-P1:LLT1 complex's structure, apart from the fact that the murine complex is symmetric while we observe two distinct binding interfaces, the primary and the secondary interaction mode of LLT1.

Despite different oligomeric forms of NKR-P1:LLT1, NKR-P1B:Clrb, and NKp65:KACL complexes, the primary receptor:ligand interaction is similar in all three cases at the level of monomer:monomer superposition - the receptor:ligand pair of monomers overlaps basically along the whole chain. The most structurally conserved regions are generally sequentially conserved beta-sheets in the core of the proteins. Figure 7 shows in red the fragments with conserved 3D position, amino acid type, and length of at least three amino acids. Such criteria are fulfilled by these fragments: in ligands - KCFYFS (in human LLT1 residues 8590), NWT (95-97), WIGL (132-135), WKW (143-145), and WICSK (182-186), and in receptors - WIGL (in human NKR-P1 residues 153-156) and ICQ (209-211). Therefore, it seems that for their interaction, the mutual orientation of the receptor and ligand scaffold is more important than the actual interaction interface.

We noted only three amino acid interaction pairs conserved in the interaction interface in at least two out of the three homologous complexes. They are gathered around residues Tyr165-Tyr171-Phe148, Arg175-Arg181-Arg158, and Tyr177-Tyr183-Phe160 of the ligand (Tab. S2). The arginine residues form equivalent hydrogen bonds in Clrb and KACL cases. In LLT1, the arginine assumes a different conformation and forms an intramolecular hydrogen bond with Asn183. The primary interaction mode in NKR-P1:LLT1 mainly relies on main chain contacts that enable fast $\mathrm{k}_{\text {on }} / \mathrm{k}_{\text {off }}$ kinetics, thus corroborating the previously published SPR-based findings ${ }^{39,40}$, and our AUC analysis. Hence, a topologically similar complex is formed in all three cases, although the underlying intermolecular mechanism of recognition is semi-independent of the actual fold and amino acid composition. However, the secondary 
interaction mode is unique to the NKR-P1:LLT1 complex and is not found in the other related complexes.
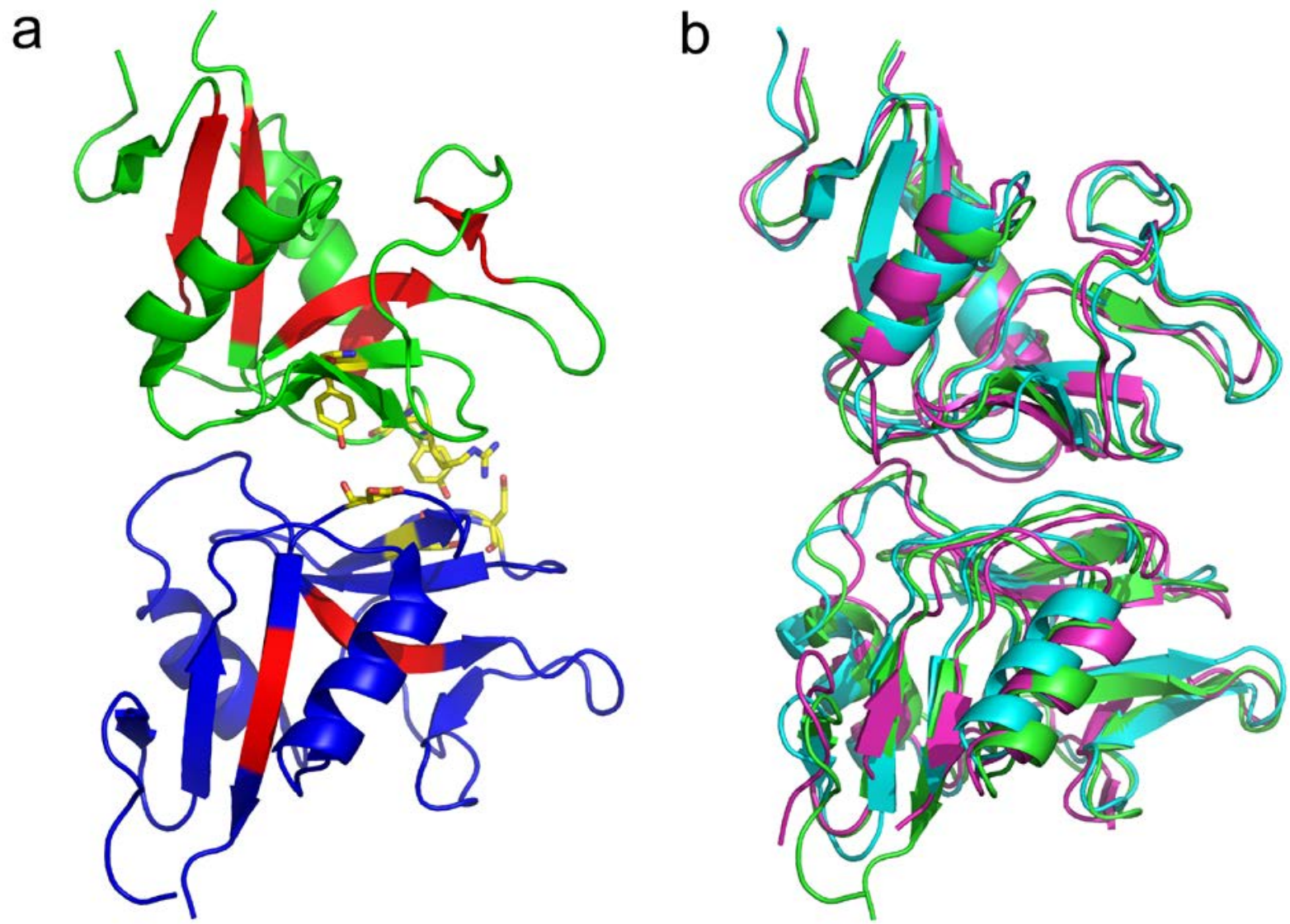

Figure 7. Anatomy of NK cell CTL receptor:ligand interactions. (a) The primary mode of interaction between human NKRP1 (blue) and LLT1 (green). The residues colored red show conserved sequence fragments at least three residues long corresponding structurally in the superposition of the three receptor:ligand interaction pairs (primary mode of human NKRP1:LLT1, murine NKR-P1B:Clrb, and human NKp65:KACL). The residues partially conserved at their interaction interface (see Tab. S2) are shown as sticks for the case of NKR-P1:LLT1. (b) Overlapped interaction pairs of the primary mode of human NKR-P1:LLT1 (green), murine NKR-P1B:Clrb (cyan), and human NKp65:KACL (magenta).

\section{Effect of receptor a1-centered dimerization on complex affinity}

Li et al. ${ }^{37}$ reported that the orientation of NKp65 bound to its ligand precludes the putative a2-centered dimerization of NKp65. Similarly, a hypothetical NKp65 a1-centered dimer is implausible as well, based on steric hindrance and the lack of stabilizing interactions. This observation contrasts with the a1-centered dimerization of NKR-P1 present in both its unbound and complexed crystal structure. Interestingly, the single-nucleotide polymorphism (SNP) c.503T>C of the human KLRB1 gene, causing the substitution of isoleucine 168 for threonine in the NKR-P1 CTLD, was reported to have a $37 \%$ frequency of the Thr168 allele $^{52}$. The authors showed that the Thr168 isoform of NKR-P1 has a lower affinity to LLT1 and a weaker inhibitory effect on NK cells. They proposed that lle168 forms a part of the interaction interface between NKR-P1 and LLT1, directly affecting LLT1 recognition by NKR- 
$P 1^{52}$. However, the structure of the NKR-P1 homodimer shows that lle168 is found at the dimerization interface rather than at the membrane-distal interaction interface - more specifically, in a small hydrophobic pocket within the dimerization interface (Fig. S3d). Therefore, we propose that the substitution of the nonpolar isoleucine residue by polar threonine caused by c.503T>C SNP indirectly affects the binding affinity because this substitution destabilizes the a1-centered NKR-P1 homodimer. Glycosylation often has a significant impact on receptor homooligomerization, as recently evidenced for, e.g., NK cell activation receptor NKp3053. NKR-P1 homodimerization is also regulated by its glycans, specifically the glycans present on Asn116 and Asn157 (Fig. S3b). Core glycan chains present at these residues contribute partially to the $\alpha 1$-centered dimerization interface, but at the same time, the glycans clash together. As a result, the stability of the $\alpha 1$-centered NKRP1 homodimer is improved by abrogating N-glycosylation on Asn157, leaving only the Asn116 glycan at the dimer interface (Fig. S4b). Interestingly, a c.470A>G SNP causing N157S mutation is also listed in the human genome variation database. However, the clinical significance of N157S mutation was not yet investigated, although it could significantly affect NKR-P1 signalization via stabilizing its ligand-bound state. The oligomeric state of the receptor might thus modulate the overall NKR-P1:LLT1 binding affinity. The NKp65:KACL complex stands out for its high affinity $\left(\mathrm{K}_{d} \sim 0.67 \mathrm{nM}\right)^{37}-\mathrm{ca} .3000 \times$ stronger than that of NKp80:AICL $\left(K_{d} \sim 2.3 \mu \mathrm{M}\right)^{9}$ and 70000-130000x than that of NKR-P1:LLT1 $\left(K_{d} \sim 48 \mu M^{39}\right.$; this study $90 \mu \mathrm{M}$ ). Due to the exceptionally high NKp65:KACL binding affinity, any putative ancestral $\alpha 1$-centered dimerization interface may have been lost in NKp65. In contrast, the NKR-P1 and NKp80 receptors may have evolved to compensate for their low affinity to their ligands by utilizing the $\alpha 1$-centered dimerization and enabling an increased avidity effect.

\section{Comparison with the previously proposed NKR-P1:LLT1 binding model}

The SPR analysis of single-residue mutants of both binding partners, performed by Kamishikiryo et al. ${ }^{39,40}$ and updated by Kita et al. ${ }^{39,40}$ based on the published LLT1 structure, identified several key residues of NKR-P1 and LLT1 essential for their interaction and proposed several pairs of interacting residues (Tab. S3). The mutated residues that had detrimental or moderate adverse effects on binding in these SPR studies are mainly found in the primary interaction interface (Fig. 1 and Tab. S3) - in LLT1: Tyr165, Asp167, Lys169, Arg175, Arg180, and Lys181, and in NKR-P1: Arg181, Asp183, Glu186, Tyr198, Tyr201, and Glu205. However, the proposed interaction pairs do not always agree with the mutual orientation of both proteins observed in the present crystal structure of the NKR-P1:LLT1 complex. For example, the proposed LLT1/NKR-P1 pairs Tyr177/Tyr198 and Arg175/Glu200 correspond well with our observed interaction pairs LLT1:Tyr177:OH/NKR-P1:Ser199:O and LLT1:Arg175:N/NKR-P1:Glu200:OE2, respectively. On the other hand, the proposed pairing 
of Glu179 from LLT1 loop L6 with Ser193 and Thr195 from NKR-P1 loop L5 only resembles the crystal structure contacts of Glu179 with Arg181 (loop L3) and Tyr198 (strand B4), located near NKR-P1 loop L5. In contrast to the SPR studies, we observed no contact between Tyr165 and Phe152, although LLT1:Tyr165 is used in the primary interface, and Phe152 is close to the NKR-P1 L0 interaction region. Lastly, we cannot confirm the suggested direct bond between LLT1:Lys169 and NKR-P1:Glu205. Although these residues are close to each other in the primary mode (the closest distance $4.3 \AA$ ), they clearly do not form a pivotal bond of the interaction interface. Furthermore, the Lys169 side chain is rather flexible, as suggested by the low quality of its electron density map in the primary mode, in contrast to all other nearby side chains with well-defined positions.

Because the secondary binding mode observed in the NKR-P1:LLT1 structure involves a different region of LLT1 than the region used in the primary binding mode, the orientation of LLT1 and NKR-P1 in this binding mode does not match the interaction pairs proposed in the SPR studies. Notwithstanding, the NKR-P1 interaction interface is very similar in primary and secondary mode; therefore, some of the previously proposed NKR-P1 interaction residues are also involved in the secondary mode (Arg181, Asp183, Tyr198, and Tyr201). Interestingly, residue LLT1:Lys169 establishes several contacts with NKR-P1 (Arg181, Ser199, and Glu200) in secondary interaction mode. However, the pair Lys169/Glu205 proposed by Kamishikiryo et al. ${ }^{39,40}$ is not observed in this mode either, and these residues are even farther apart - ca $11 \AA$. The presence of Lys169 in both primary and secondary interaction interfaces of LLT1 suggests that this residue plays an important role in the overall complex formation. Accordingly, the reported Lys169Glu mutation of LLT1 ${ }^{39}, 40$ would lead to a co-localization of several negative side chains in the secondary interface (Glu200 and Asp183 of NKR-P1, and mutated Glu169 of LLT1), thus indicating that the disruption of the NKR-P1:LLT1 interaction observed in the previous SPR experiments most likely resulted from the weakening of the secondary rather than the primary interface. Kamishikiryo et al. ${ }^{39}$, ${ }^{40}$ subsequently restored binding by introducing the Glu205Lys mutation in NKR-P1. Based on our structure, this effect would be explained by the strengthening of the primary interface. Thus, the previously published SPR-based interaction data largely agree with the interaction modes observed between NKR-P1 and LLT1 in the present crystal structure, although some previously suggested interaction pairs were misassigned and are not present in either interaction interface.

\section{Assembly of the NKR-P1:LLT1 complex on the cell surface}

Several authors have previously suggested an avidity effect of multimerization upon the interaction that compensates for the low affinity of the NKR-P1:LLT1 complex ${ }^{37,40,47 .}$ Interestingly, in the present structure of this complex, we indeed observe the formation of a 
chain of repeating NKR-P1 and LLT1 homodimers. This pseudo-linear multimer has a zigzag shape in which the membrane-proximal parts of the two proteins are on opposite sides (Fig. 8), and it is structurally based on the alternating helix $\alpha 1 / \alpha 2$-centered NKR-P1/LLT1 homodimers (the chain-forming effect) and the simultaneous involvement of both primary and secondary interaction modes (steric effect). On the contrary, an artificially constructed multimeric model of NKR-P1:LLT1 engaged in just the primary mode shows a chain of homodimers with a non-linear, almost helical conformation (Fig. S7a). Furthermore, the NKR-P1 stalk regions sterically clash, and the LLT1 stalk regions are exposed outside the complex core in many different directions. Thus, such multimer is unlikely compatible with cell membrane anchoring and formation within the immune synapse. NKR-P1:LLT1 engaged in just the secondary mode would also form a helical multimer with both receptor and ligand stalk regions pointing in many different directions outside the complex core (Fig. S7b). At the same time, when bound to LLT1 dimer in both primary and secondary interaction mode, the neighboring NKR-P1 molecules create a mutual accessory contact of not an insignificant energetic contribution to the stability of the whole assembly (Fig. 3a, marked with a black star). This accessory contact encompasses a similar number of hydrogen bonds and size as the secondary binding interface between NKR-P1 and LLT1 itself (Fig. S8), and it could thus be considered an additional stabilizing element favoring a combination of the primary and secondary binding modes rather than a single one of them.

To determine whether such zipper-like clusters of NKR-P1:LLT1 occur not only in crystal and to some extent in solution (Fig. 4 and S6) but also on the cell surface, we used singlemolecule localization microscopy to examine full-length NKR-P1 transfectants labeled with anti-NKR-P1 Alexa-647 mAb in the presence or absence of LLT1 and LLT1 ${ }^{\text {SIM }}$ (Fig. 5). We observed a significant increase in NKR-P1 cluster size and area upon the addition of soluble LLT1, but not its secondary interaction mode mutant, LLT1 ${ }^{\text {SIM }}$. Additionally, our SEC-SAXS data were best-fit with multimer chains in alternating primary and secondary interaction modes (Fig. S6), even though the artificial test models discussed above were included in the analysis as well. Acceptable $X^{2}$ values were obtained primarily for multimer models containing the alternating arrangement. Taken together, we conclude that combining both interaction modes is necessary for a biologically plausible multimeric interaction, as further supported by the NK cell-mediated cytotoxicity assay results (Fig. 6). 
a

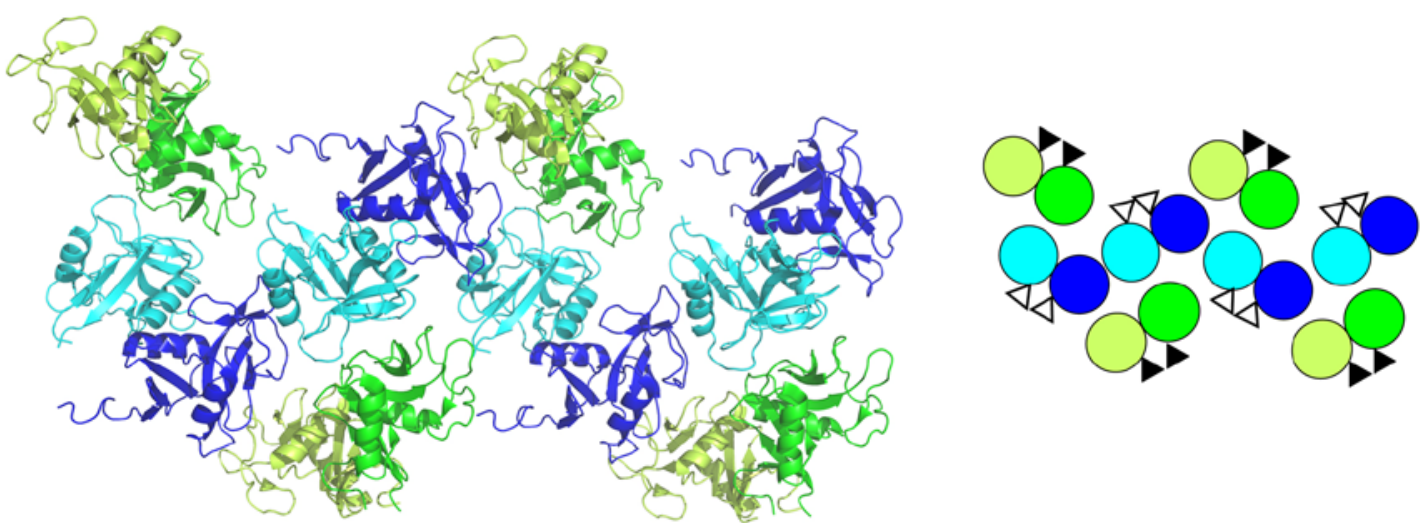

b

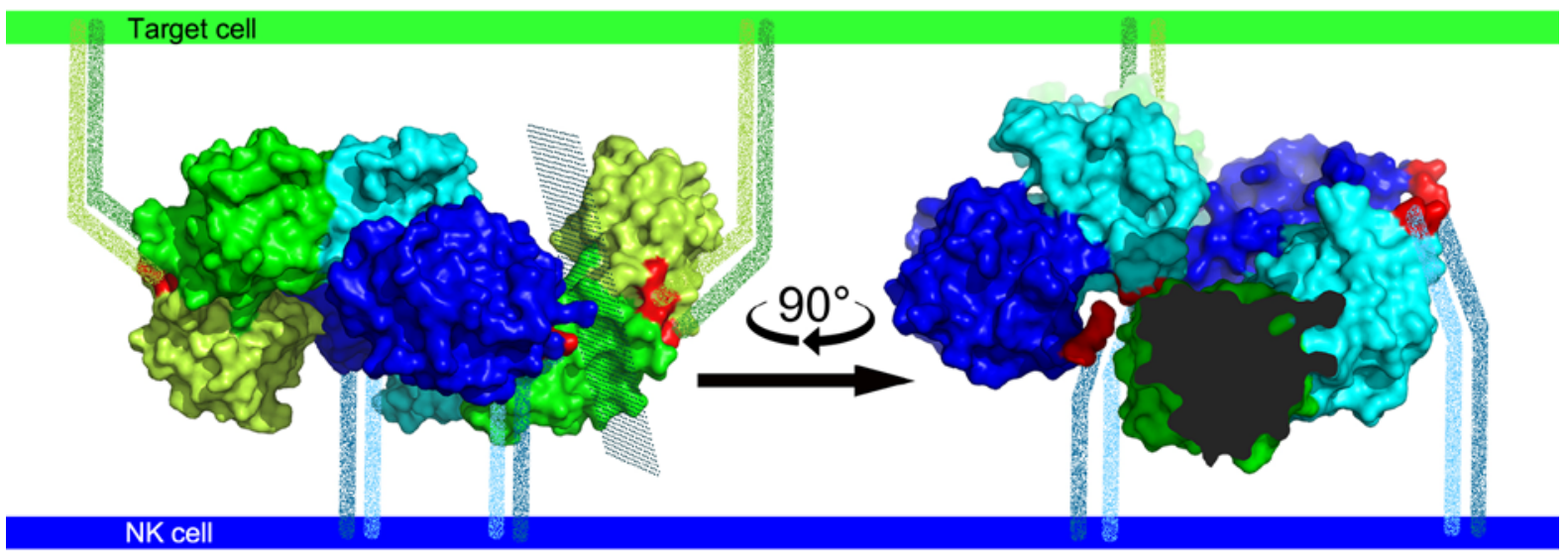

C NKR-P1 dimer
conformation change

\section{Standard interaction model}

Proposed interaction model

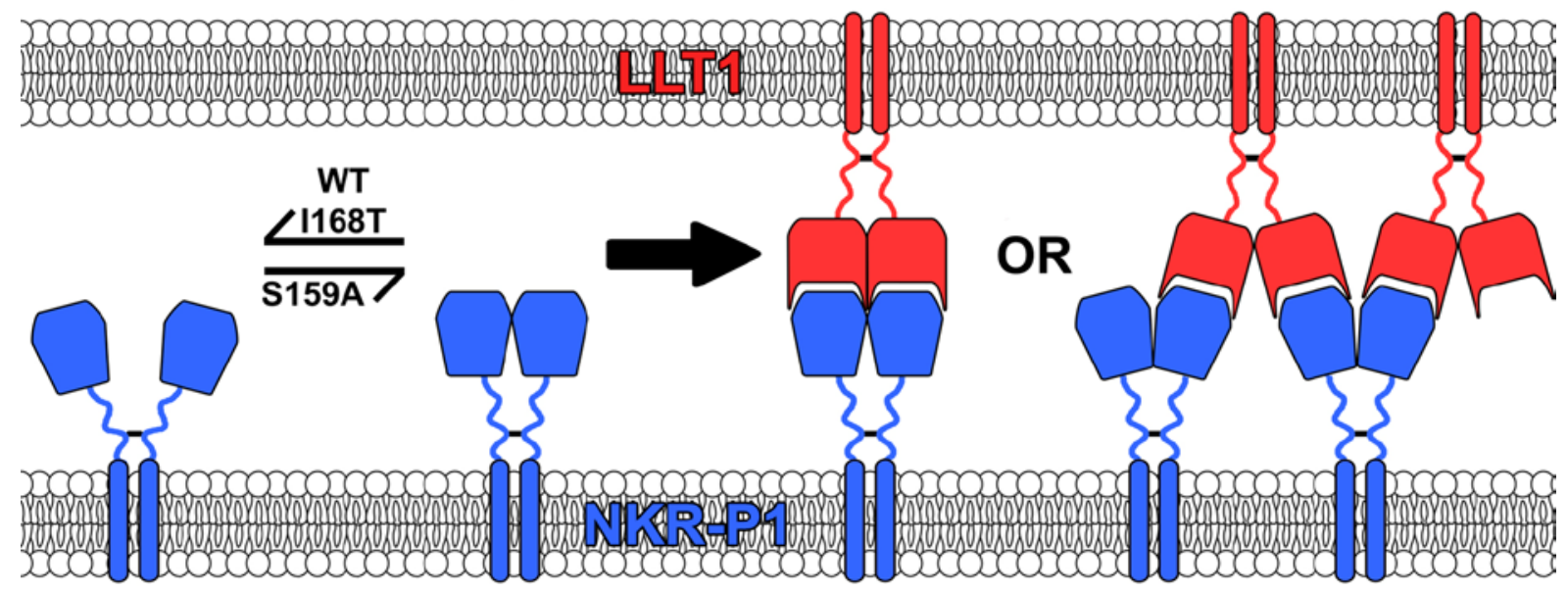

Figure 8. Organization of the NKR-P1:LLT1 complexes on the cell surface. (a) Representation of four adjacent asymmetric units within the NKR-P1:LLT1 complex crystal, excluding the additional unrelated NKR-P1 dimer. The NKR-P1 (blue and cyan) and LLT1 (green and lemon) dimers alternate in primary (cyan and green) and secondary (blue and lemon) interactions, forming a chain-like structure. The schematic depiction of this arrangement is shown in the inset with the same color code. The black and white triangles represent $\mathrm{N}$-termini positions, pointing behind and in front of the display plane, respectively. (b) Depiction of the hypothetical arrangement of the chain-like structure upon contact of an NK cell (bottom, blue) with a target cell (top, green) showing the crystal structure of two NKR-P1 dimers (cyan and blue) interacting with two LLT1 dimers (green and lemon) in the primary (cyan and green) and secondary (blue and lemon) modes. The first three N-terminal residues in the structures are highlighted in red. The flexible stalk regions connecting the $\mathrm{N}$-termini and cell membranes are represented as 
speckled lines of the corresponding color-coding. The view on the right-hand side is clipped for clarity at the plane indicated on the left-hand side view. (c) Schematic depiction of NKR-P1 extracellular domain dynamics and possible ligand binding arrangements. NKR-P1 is expressed as a disulfide-linked homodimer; however, its CTLDs may undergo conformation change similar to monomer-dimer equilibrium. Such putative equilibrium would be shifted towards monomeric species for the wild-type protein and its I168T allelic variant ${ }^{52}$, while a dimeric arrangement corresponding to the non-covalent dimer observed in the herein described crystal structures would be promoted for the S159A variant (left-hand side). Such NKR-P1 dimer could then interact with the cognate LLT1 ligand (itself being expressed as a disulfide-linked homodimer as well and forming stable noncovalent dimers with its CTLDs) in the previously suggested standard model of NK cell receptor - CTL ligand interaction (middle) or alternate with the dimeric ligand in the proposed chain-like arrangement based on NKR-P1:LLT1 complex crystal structure (right-hand side).

Albeit such functional multimerization of NK CTLRs has been mostly overlooked, the formation of similar nanoclusters is well described for interaction between the immunoglobulin family of KIRs and MHC class I glycoproteins ${ }^{54}$ or for interaction between KIR2DL1 and NKG2D ${ }^{55}$. Moreover, a periodic zipper-like network of interacting dimers was reported in the crystal structures of the co-stimulatory immunocomplexes B7-1:CTLA-4 and B7-2:CTLA-4 ${ }^{56,57}$. Interestingly, B7-1 is expressed on the cell surface in a dynamic equilibrium between monomers and non-covalent dimers. Upon interaction with the costimulatory receptor CD28, B7-1 forms an interaction network composed of B7-1 and CD28 homodimers. The uncoupling of this interaction is facilitated by B7-1 dissociation to monomers, whereas insertion of B7-1 obligate dimer leads to prolonged, abnormal signaling between antigen-presenting cells and $\mathrm{T}$ cells ${ }^{58}$.

Although as full-length proteins, both LLT1 and NKR-P1 form covalent disulfide homodimers on cell surface ${ }^{7,36}$, to our knowledge, the dimeric state of their CTL ectodomains has not yet been assessed in live cells. Conversely, the formation of the helix $\alpha 2$-centered non-covalent homodimer of soluble LLT1 ectodomain has been previously characterized ${ }^{40,43,44}$ and likely occurs within the full-length protein as well. The human NKR-P1 helix a1-centered dimer uses fewer intermolecular contacts and has a smaller contact surface area than helix $\alpha 2$ centered dimeric CTLRs and is thus less stable. Nevertheless, its formation would expectedly increase in the context of the full-length NKR-P1 receptor disulfide homodimer. At the same time, the length of the NKR-P1 stalk region (25 amino acids) confer enough flexibility for CTLD monomer/dimer equilibrium within the disulfide homodimer of the fulllength receptor itself, which would be then further regulated by polymorphism and glycosylation heterogeneity at the NKR-P1 dimerization interface (Fig. 8c, bottom left). Thus, similarly to the B7-1:CD28 system, an equilibrium between the monomeric and dimeric states of CTL ectodomains, modifying and fine-tuning the ability of NKR-P1 to form stable higher-order complexes with LLT1, may consequently regulate the strength and signaling of the NKR-P1:LLT1 system, while cross-linking of NKR-P1 by LLT1 within the immune 
synapse may provide enough avidity for stable signal transduction by this low-affinity interaction complex.

In conclusion, presented data altogether show that the crystal structure of the NKR-P1:LLT1 complex constitutes a novel way of C-type lectin-like NK cell receptor:ligand multimerization on the cell surface that explains how ligand binding overcomes low affinity through receptor cross-linking within the immune synapse.

\section{Acknowledgments}

This study was supported by BIOCEV (ERDF CZ.1.05/1.1.00/02.0109 and CZ.02.1.01/0.0/0.0/16_013/0001776), the Czech Science Foundation (15-15181S, 1810687S), the Ministry of Education, Youth and Sports of the Czech Republic (LG14009 and LM2015043 CIISB for CMS Biocev; LTC17065 in the frame of the COST Action CA15126 MOBIEU), the Charles University (SVV 260427/2020, GAUK 161216 and 1378219), and BioStruct-X (EC FP7 project 283570). Microscopy was performed in the Laboratory of Confocal and Fluorescence Microscopy (ERDF CZ.1.05/4.1.00/16.0347 and CZ.2.16/3.1.00/21515) supported by the Czech-Biolmaging (LM2015062). The authors wish to thank Dr. Carlos V. Melo for critically proofreading the manuscript. The authors also acknowledge the support and the use of resources of Instruct-ERIC through the R\&D pilot scheme APPID 56 and 286. The Wellcome Trust Centre for Human Genetics is supported by the Wellcome Trust (grant 090532/Z/09/Z). We thank Diamond Light Source for beamtime (proposal MX10627) and the staff of beamlines 103 and 21 for assistance with data collection.

\section{Author contributions}

$\mathrm{JB}, \mathrm{OS}, \mathrm{BK}, \mathrm{SP}, \mathrm{EP}$, and CA contributed to protein expression and purification; JB and $\mathrm{YZ}$ performed the protein crystallization; $\mathrm{YZ}$ and $\mathrm{KH}$ performed the $\mathrm{X}$-ray diffraction measurements; JB, TS, JS, TK, JDu, and JDo contributed to the data processing and model refinement; OS performed the SEC-SAXS data measurement; JB and TS performed the SAXS data analysis; OV performed the analytical ultracentrifugation measurements and analysis; JB and BK acquired and analyzed the dSTORM data; BK and DC performed the NK cytotoxicity assay; JB, TS, JDo, and OV designed the experiments and wrote the manuscript with critical input from $\mathrm{JH}$.

\section{Competing financial interests}

The authors declare no competing financial interests. 


\section{Methods}

\section{Protein expression and purification}

Stabilized H176C form of the soluble LLT1 ectodomain (GIn72-Val191) was transiently expressed in HEK293S GnTl- cells, as previously described ${ }^{44}$. The N120R, R153E, K169A secondary interaction mode mutant LLT1 ${ }^{\mathrm{SIM}}$ was cloned and produced in the same way. The C-type lectin-like domain of human NKR-P1 was produced similarly in stably transfected HEK293S $\mathrm{GnTl}^{-}$cells ${ }^{51}$. Briefly, the expression construct corresponding to the extracellular CTL domain of NKR-P1 (Gly90-Ser225) was subcloned into the pOPINGGTneo plasmid (kindly provided by Prof. Ray Owens, University of Oxford), flanked by the N-terminal secretion leader and by the C-terminal His-tag (with the ETG and the $\mathrm{KHHHHHH}$ at the $\mathrm{N}$ and C-termini of the secreted protein, respectively). Suspension culture of HEK293S $\mathrm{GnTI}^{-}$ cells ${ }^{59}$ was transfected with a 1:3 (w/w) mixture of the expression plasmid and 25-kDa linear

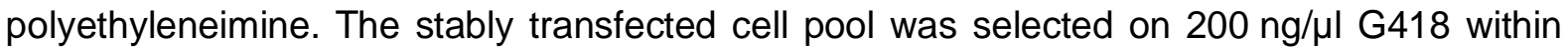
three weeks. The secreted proteins were purified from the harvested media by two-step chromatography - an IMAC was performed on a Talon column (GE Healthcare), followed by SEC on a Superdex 200 10/300 GL (GE Healthcare) in 10 mM HEPES pH 7.5 with 150 mM $\mathrm{NaCl}$ and $10 \mathrm{mM} \mathrm{NaN}_{3}$. For deglycosylation, GST-fused Endo $\mathrm{F}^{60}$ was added in a 1:100 weight ratio to proteins in SEC buffer with $50 \mathrm{mM}$ citrate $\mathrm{pH} 5.5$ and incubated for 2 hours at $37^{\circ} \mathrm{C}$. The deglycosylated proteins were then purified by batch affinity chromatography on Glutathione Sepharose 4B resin (GE Healthcare) followed by SEC, as described above.

\section{Crystallization}

NKR-P1 glycosylated (structure NKR-P1_glyco) - Soluble human NKR-P1 ectodomain at $20 \mathrm{mg} / \mathrm{ml}$ in SEC buffer was crystallized using the sitting drop vapor diffusion method. Drops (100 $\mathrm{nl}$ of reservoir solution and $100 \mathrm{nl}$ of protein solution) were set up using a Cartesian Honeybee 961 robot (Genomic Solutions) at $294 \mathrm{~K}$. The reservoir consisted of $20 \% \mathrm{w} / \mathrm{v}$ PEG 3350, 200 mM di-sodium tartrate pH 7.2 (PEG/Ion screen, condition 36; Hampton Research). A hexagonal crystal with dimensions of $150 \times 150 \times 20 \mu \mathrm{m}$ was cryoprotected by soaking in the reservoir solution with the addition of $25 \%(\mathrm{v} / \mathrm{v})$ ethylene glycol.

NKR-P1 deglycosylated (structure NKR-P1_deglyco) - The Endo F1-deglycosylated soluble human NKR-P1 ectodomain was concentrated to $12 \mathrm{mg} / \mathrm{ml}$ and crystallized as described above. The reservoir consisted of 20\% w/v PEG 3350, $200 \mathrm{mM}$ ammonium fluoride, and 200 mM lithium chloride pH 6.2 (PEG/Ion screen, condition 3, Additive screen, condition 17; Hampton Research). A $50 \times 50 \times 150 \mu \mathrm{m}$ rod-shaped crystal was cryoprotected as above by adding 25\% (v/v) glycerol. 
NKR-P1:LLT1 complex (structure NKR-P1:LLT1) - The Endo F1-deglycosylated soluble human NKR-P1 and LLT1 ectodomains were mixed at a 1:1 molar ratio and concentrated to $8 \mathrm{mg} / \mathrm{ml}$ of total protein concentration. The protein complex was crystallized as described above; drops (200 $\mathrm{nl}$ of the reservoir and $100 \mathrm{nl}$ of protein solutions) were seeded with $50 \mathrm{nl}$ of stock solution of crushed needle-shaped crystals of deglycosylated NKR-P1 grown in 20\% w/v PEG 3350, 200 mM ammonium fluoride pH 6.2 (PEG/Ion screen, condition 3; Hampton Research). The reservoir consisted of $200 \mathrm{mM}$ ammonium sulfate, 20\% w/v PEG MME 5000, 100 mM Tris pH 7.5 (Proplex screen, condition 1-40; Molecular Dimensions). A tetragonal bipyramid crystal of dimensions $30 \times 30 \times 80 \mu \mathrm{m}$ was cryoprotected as detailed above by adding $25 \%$ v/v glycerol.

\section{Data collection}

All diffraction data were collected at the Diamond Light Source (Harwell, UK) at beamline I03 using a wavelength of $0.97625 \AA$ and a PILATUS3 6M detector. The crystal-detector distance was set to $340 \mathrm{~mm}$, exposure time per image was $0.02 \mathrm{~s}$, oscillation width was $0.1^{\circ}$, and the temperature was $100 \mathrm{~K} .7200$ images were collected for each dataset. In the case of the NKR-P1:LLT1 complex, only 5000 images were finally used for data processing.

\section{Data processing and structure solution}

All diffraction images were indexed and integrated using the XDS package ${ }^{61}$, scaled using AIMLESS $^{62}$, and $5 \%$ of randomly selected reflections were used as an $R_{\text {free }}$ set. The phase problem was solved by molecular replacement - NKR-P1_glyco: in program BALBES ${ }^{63}$ using the structure of the human NK cell receptor KLRG1 bound to E-cadherin (PDB ID 3FF7 ${ }^{64}$ ); NKR-P1_deglyco: 6 chains found in PHASER ${ }^{65}$ using murine NKR-P1A (PDB ID $\left.3 T 3 A^{66}\right)$ were completed with 2 chains found in MOLREP ${ }^{67}$; NKR-P1:LLT1: 4 chains found in BALBES as NKR-P1 chains (using the structure of murine dectin-1, PDB ID 2BPD ${ }^{49}$ ) were completed with two more chains in MOLREP, and all 6 chains were manually reinterpreted as 4 NKR-P1 chains and as 2 LLT1 chains. Refinement was performed using REFMAC5 ${ }^{68}$ and by manual editing in $\mathrm{COOT}^{69}$. The last cycle of refinement was performed using all reflections. The final data processing and structure parameters are outlined in Tab. 1.

\section{Structure quality assessment}

NKR-P1_glyco - The structure, comprising one dimer of the glycosylated human NKR-P1 CTLD, is overall well defined in the electron density map, corresponding to the high resolution of the structure $(1.8 \AA)$. Glycosylation at the dimerization interface does not show the overlapping features observed in the structures of deglycosylated NKR-P1 (below). GIcNAc was modeled on Asn116 with full occupancy in both chains, whereas glycosylation 
at Asn157 was not observed in the electron density map. All modeled glycosylation chains (GlcNAc $\mathrm{Man}_{5}$ at A/Asn169, GlcNAc $\mathrm{Man}_{3}$ at B/Asn169, and GlcNAc at residues Asn116 in both chains) are well localized in the electron density map.

NKR-P1_deglyco - The asymmetric unit comprises four dimers of the human NKR-P1 CTLD deglycosylated after the first GIcNAc unit. The length of the localized part of the protein chain varies from the shortest chains A, F, and G, with modeled residues Leu91-Leu214, to the longest chain $\mathrm{H}$, with residues Gly90-Arg218. GlcNAc at residue Asn169 is well localized, while GICNAc units bound to Asn116 and Asn157 at the dimerization interface are present in alternative and overlapping positions; residue Asn116 also shows alternative conformers. GlcNAc units bound to Asn157 and Asn116 were modeled with 0.5 occupancies, and only the most distinct units from each overlapping pair were modeled (Tab. 1).

NKR-P1:LLT1 - The asymmetric unit contains two dimers of the human NKR-P1 CTLD and one dimer of the LLT1 CTLD. The structure has a well-defined electron density map, and all protein chains can be unambiguously assigned. The most distinct difference peaks correspond to non-interpretable small ligands. LLT1 has well-localized GICNAC units at residues Asn95 and Asn147. Localized GIcNAc units of NKR-P1 are the same as those identified in the NKR-P1_deglyco structure (previous paragraph).

\section{PDB deposition}

The crystal structures were deposited in the Protein Data Bank under the codes 5MGR (NKR-P1_glyco), 5MGS (NKR-P1_deglyco), and 5MGT (NKR-P1:LLT1).

\section{CD spectroscopy}

Circular dichroism (CD) spectra of wild-type and S159A NKR-P1 and wild-type and SIM LLT1 were recorded using a Chirascan Plus CD spectropolarimeter (Applied Photophysics) and a $0.1 \mathrm{~cm}$ pathlength quartz cell. Spectra were recorded over the wavelength range of 195-260 nm in steps of $1 \mathrm{~nm}$ at room temperature. The sample concentrations were $0.2 \mathrm{mg} / \mathrm{ml}$ and $0.3 \mathrm{mg} / \mathrm{ml}$ for NKR-P1 and LLT1 protein samples, respectively; in $10 \mathrm{mM}$ HEPES, $150 \mathrm{mM} \mathrm{NaCl}$ pH 7.5 sample buffer. The CD signal was expressed as ellipticity, and the resulting spectra were buffer subtracted. Secondary structure composition was analyzed using the CDNN program provided with the Chirascan Plus CD spectropolarimeter.

\section{Analytical ultracentrifugation}

NKR-P1:LLT1 complex formation and dimerization of NKR-P1 S159A mutant were analyzed in an analytical ultracentrifuge ProteomeLab XL-I equipped with an An-50 Ti rotor (Beckman Coulter, USA $)^{70}$. For the sedimentation velocity experiment, samples of glycosylated NKRP1, LLT1, and their equimolar mixtures with increasing concentrations in SEC buffer were 
spun at $48000 \mathrm{rpm}$ at $20^{\circ} \mathrm{C}$, and 150 scans with $0.003 \mathrm{~cm}$ spatial resolution were recorded in 5-min steps using absorbance optics. Buffer density and protein partial specific volumes were estimated in SEDNTERP (www.jphilo.mailway.com). Data were analyzed with SEDFIT ${ }^{71}$ using the continuous $\mathrm{c}(\mathrm{s})$ distribution model. Binding isotherms (and figures illustrating $A \cup C$ data) were prepared in $\mathrm{GUSSI}^{72}$ and then best-fit in SEDPHAT ${ }^{73}$ using hetero-association models $A+B \Leftrightarrow A B$ or $A+B \Leftrightarrow A B+B \Leftrightarrow A B B$, where $A$ is the LLT1 dimer and $B$ is NKR-P1 monomer, respectively. Only $K_{D}$ and sedimentation coefficients of $A B$ or $A B B$ were floated in the fit; the other parameters were kept constant at known values.

\section{Microscale thermophoresis}

For the microscale thermophoresis (MST) measurements of NKR-P1:LLT1 and NKRP1:LLT1 ${ }^{\text {SIM }}$ interactions, the NKR-P1 has been fluorescently labeled with Atto 488 NHS-ester (Merck) at $\mathrm{pH} \mathrm{6.5;} \mathrm{the} \mathrm{excess} \mathrm{label} \mathrm{was} \mathrm{removed} \mathrm{by} \mathrm{size} \mathrm{exclusion} \mathrm{chromatography.}$ Fluorescently labeled 100 nM NKR-P1 was mixed with dilution series of LLT1 or LLT1 ${ }^{\text {SIM }}$ and analyzed in standard capillaries in an NT.115 Monolith (NanoTemper) using 30\% LED excitation power and 60\% MST power. Raw data were analyzed in PALMIST ${ }^{74}$; the exported isotherms were best-fit in SEDPHAT ${ }^{73}$ and figures prepared in $\mathrm{GUSSI}^{72}$.

\section{Small-angle $X$-ray scattering}

SEC-SAXS data for the NKR-P1:LLT1 complex were collected at the Diamond Light Source (Didcot, UK) at beamline 21 using an Agilent $1200 \mathrm{HPLC}$ system with $2.4 \mathrm{~mL}$ Superdex 200 column (GE Healthcare), a Pilatus P3-2M detector, $12.4 \mathrm{keV}$ radiation, and $4.014 \mathrm{~m}$ sampleto-detector distance. The human NKR-P1 and LLT1 ectodomains with GICNAC $2 M^{2}$ glycans $_{5}$ diluted in $10 \mathrm{mM}$ HEPES, $150 \mathrm{mM} \mathrm{NaCl}, 10 \mathrm{mM} \mathrm{NaN}_{3}$, pH 7.5 were mixed at a 1:1 molar ratio. The data were collected at $293 \mathrm{~K}$ for buffer and protein samples at a $15 \mathrm{mg} / \mathrm{ml}$ loading concentration. The data in selected intervals (frames 355-367, 368-378, 379-388, 388-399, 431-440, 481-491) were solvent-subtracted in SCÅTTER (developed by Robert Rambo at the Diamond Light Source, http://www.bioisis.net/tutorial/9) and merged and characterized using the ATSAS ${ }^{75}$ package. As proof of the data quality, the scattering plot, Kratky plot, and pair distance distribution function $P(r)$ are shown in Fig. 4 for intervals 389-399 (a sample data range from the first peak) and 481-491 (a sample data range from the second peak). The scattering plot and Guinier plot for all the data ranges are shown in Fig. S6.

Using the $P(r)$ pair distance distribution functions, twenty ab initio structure models were calculated with DAMMIF 2.7.2 ${ }^{76}$ for each data interval. The models were compared using the DAMSEL command and averaged using DAMAVER ${ }^{77}$. The final models were visualized in CHIMERA $^{78}$ as envelopes $15 \AA$ above the beads. Agreement between the SAXS data and our 3D crystal structure of the NKR-P1:LLT1 complex was evaluated using OLIGOMER ${ }^{79}$. All 
possible multimers of NKR-P1:LLT1 assemblies were considered (see the Results section and Fig. S6).

\section{Super-resolution microscopy}

dSTORM microscopy - Full-length NKR-P1 stable transfectants were generated in HEK293S $\mathrm{GnTl}^{-}$cell line using the piggyBac transposon-based system with doxycyclineinducible protein expression ${ }^{80}$. Microscopy samples were prepared from cells treated with $5 \mathrm{ng} / \mathrm{ml}$ doxycycline overnight. Cells were washed with PBS and incubated with $8.4 \mathrm{mg} / \mathrm{ml}$ LLT1 or LLT1 ${ }^{\text {SIM }}$, or PBS, for 1 hour at $37^{\circ} \mathrm{C}$. After incubation, cells were allowed to settle on the surface of PLL-coated slides for 25 min at $37^{\circ} \mathrm{C}$. Cells were then fixed with 4\% PFA and $0.2 \%$ GA for $10 \mathrm{~min}$ at room temperature and washed three times with PBS. For antibody staining, cells were first blocked with 5\% BSA for 30 min and stained with Alexa Fluor ${ }^{\circledR} 647$ anti-human CD161 antibody (clone HP-3G10; BioLegend) at $10 \mu \mathrm{g} / \mathrm{ml}$ in blocking solution for 60 min. Samples were washed five times with PBS before post-fixation with 4\% PFA and $0.2 \% \mathrm{GA}$ for $10 \mathrm{~min}$. Finally, cells were treated with $15 \mathrm{mM} \mathrm{NH}_{4} \mathrm{Cl}$ and washed two times with PBS.

dSTORM images were acquired with Carl Zeiss Elyra PS.1 in the Laboratory of Confocal and Fluorescence Microscopy (Faculty of Science, Charles University). Fiducial markers were added to the buffer and allowed to settle on the sample for 1 hour. Before measurement, the buffer was exchanged to glucose oxidase/catalase/MEA-based imaging buffer and sealed with cover glass and silicon to prevent buffer oxidation. For each cell, $2 \times 10^{4}$ raw images were acquired in HP TIRF illumination mode with an exposure time of $15 \mathrm{~ms}$, using $100 \%$ of $642 \mathrm{~nm}$ laser power and $100 \times, 1.46$ numerical aperture, oil immersion objective.

Image data analysis - Super-resolution dSTORM images were reconstructed from raw image sequences with the ThunderSTORM plug-in ${ }^{81}$ for the ImageJ processing software ${ }^{82}$. Sub-pixel localization of molecules was performed by fitting an integrated Gaussian pointspread function models using the maximum likelihood estimation fitting method ${ }^{83}$. Reconstructed dSTORM images were corrected for drift using fiducial markers and for multiple localizations from a single source by merging events within $20 \mathrm{~nm}$ appearing in subsequent frames (with an off-gap of 5 frames).

Voronoi tessellation cluster analysis was performed in ClusterViSu ${ }^{84}$ on a whole inner cell surface. A threshold value of 250 was chosen by comparing the distribution of Voronoi polygon surface values between localizations in experimental regions and randomized regions of the same density of events. Clusters containing less than two events were discarded from the data set. 
Statistical analysis - Multiple means were compared with one-way analysis of variance (ANOVA) with Bonferroni correction in OriginPro 2018 (v. b9.5.1.195). Graphs show mean values, and error bars represent the SD. Values of $p>0.05$ are indicated as not significant; statistically significant $p$ values are indicated with asterisks $\left({ }^{*} p<0.05,{ }^{* *} p<0.01\right.$,

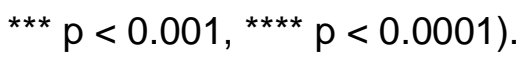

\section{NK cell-mediated cytotoxicity assay}

The influence of LLT1 or LLT1 ${ }^{\text {SIM }}$ on inhibition of the cytotoxic activity of primary NK cells was assessed by flow cytometry. Primary NK cells were isolated by negative selection using an NK cell isolation kit (Miltenyi Biotec) according to the manufacturer's protocol. Purified NK cells were cultured overnight at $1 \times 10^{6} \mathrm{cells} / \mathrm{ml}$ in RPMI 1640 supplemented with $10 \%$ FCS, 100 units $/ \mathrm{ml}$ penicillin, $100 \mu \mathrm{g} / \mathrm{ml}$ streptomycin, and $80 \mathrm{ng} / \mathrm{ml} \mathrm{IL-2}$ (Sigma-Aldrich) for their activation. K562 target cells were stained with CellTrace Violet Proliferation Kit (Thermo Fisher). Following the staining, $1 \times 10^{4}$ target cells were mixed with activated NK cells in a 40:1 (E:T) ratio. Various concentrations of LLT1 protein variants were added, keeping the final reaction volume at $20 \mu \mathrm{l}$. After 4 hours of incubation, the cell mixture was centrifuged at $300 \times \mathrm{g}$ and stained with $1 \mu \mathrm{g} / \mathrm{ml} 7$-AAD. Cells were analyzed using a BD LSR II flow cytometer (BD Biosciences). Three independent cytotoxicity assays using NK cells from three different healthy donors were performed in triplicates (or duplicates if PBMC fraction did not provide enough NK cells). Data are presented as a mean of measurements with SD. When applicable, data were statistically evaluated by one-way ANOVA with values of $p<$ 0.05 considered statistically significant.

\section{References}

1. Vivier, E. et al. Innate or adaptive immunity? The example of natural killer cells. Science 331, 44-49 (2011).

2. Cerwenka, A. \& Lanier, L.L. Natural killer cell memory in infection, inflammation and cancer. Nature reviews. Immunology 16, 112-123 (2016).

3. Yokoyama, W.M. \& Plougastel, B.F. Immune functions encoded by the natural killer gene complex. Nature reviews. Immunology 3, 304-316 (2003).

4. Bartel, Y., Bauer, B. \& Steinle, A. Modulation of NK cell function by genetically coupled C-type lectin-like receptor/ligand pairs encoded in the human natural killer gene complex. Frontiers in immunology 4, 362 (2013).

5. Zelensky, A.N. \& Gready, J.E. The C-type lectin-like domain superfamily. The FEBS journal 272, 6179-6217 (2005). 
6. Rozbesky, D. et al. Re-evaluation of binding properties of recombinant lymphocyte receptors NKR-P1A and CD69 to chemically synthesized glycans and peptides. International journal of molecular sciences 15, 1271-1283 (2014).

7. Lanier, L.L., Chang, C. \& Phillips, J.H. Human NKR-P1A. A disulfide-linked homodimer of the C-type lectin superfamily expressed by a subset of NK and T lymphocytes. Journal of immunology 153, 2417-2428 (1994).

8. Spreu, J. et al. Interaction of C-type lectin-like receptors NKp65 and KACL facilitates dedicated immune recognition of human keratinocytes. Proceedings of the National Academy of Sciences of the United States of America 107, 5100-5105 (2010).

9. Welte, S., Kuttruff, S., Waldhauer, I. \& Steinle, A. Mutual activation of natural killer cells and monocytes mediated by NKp80-AICL interaction. Nature immunology 7, 1334-1342 (2006).

10. Vogler, I. \& Steinle, A. Vis-a-vis in the NKC: genetically linked natural killer cell receptor/ligand pairs in the natural killer gene complex (NKC). Journal of innate immunity 3, 227-235 (2011).

11. Aldemir, H. et al. Cutting edge: lectin-like transcript 1 is a ligand for the CD161 receptor. Journal of immunology 175, 7791-7795 (2005).

12. Rosen, D.B. et al. Cutting edge: lectin-like transcript-1 is a ligand for the inhibitory human NKR-P1A receptor. Journal of immunology 175, 7796-7799 (2005).

13. Poggi, A., Costa, P., Tomasello, E. \& Moretta, L. IL-12-induced up-regulation of NKRP1A expression in human NK cells and consequent NKRP1A-mediated down-regulation of NK cell activation. European journal of immunology 28, 1611-1616 (1998).

14. Exley, M., Porcelli, S., Furman, M., Garcia, J. \& Balk, S. CD161 (NKR-P1A) costimulation of CD1d-dependent activation of human $T$ cells expressing invariant $V$ alpha $24 \mathrm{~J}$ alpha $\mathrm{Q} \mathrm{T}$ cell receptor alpha chains. The Journal of experimental medicine 188, 867-876 (1998).

15. Ussher, J.E. et al. CD161++ CD8+ T cells, including the MAIT cell subset, are specifically activated by IL-12+IL-18 in a TCR-independent manner. European journal of immunology 44, 195-203 (2014).

16. Fergusson, J.R. et al. CD161 defines a transcriptional and functional phenotype across distinct human T cell lineages. Cell reports 9, 1075-1088 (2014).

17. Germain, C. et al. Induction of lectin-like transcript 1 (LLT1) protein cell surface expression by pathogens and interferon-gamma contributes to modulate immune responses. The Journal of biological chemistry 286, 37964-37975 (2011). 
18. Bennett, I.M. et al. Definition of a natural killer NKR-P1A+/CD56-/CD16- functionally immature human NK cell subset that differentiates in vitro in the presence of interleukin 12. The Journal of experimental medicine 184, 1845-1856 (1996).

19. Cosmi, L. et al. Human interleukin 17-producing cells originate from a CD161+CD4+ T cell precursor. The Journal of experimental medicine 205, 1903-1916 (2008).

20. Mathewson, N.D. et al. Inhibitory CD161 receptor identified in glioma-infiltrating T cells by single-cell analysis. Cell 184, 1281-1298 e1226 (2021).

21. Poggi, A., Costa, P., Zocchi, M.R. \& Moretta, L. Phenotypic and functional analysis of CD4+ NKRP1A+ human T lymphocytes. Direct evidence that the NKRP1A molecule is involved in transendothelial migration. European journal of immunology 27, 2345-2350 (1997).

22. Chalan, P. et al. Expression of Lectin-Like Transcript 1, the Ligand for CD161, in Rheumatoid Arthritis. PloS one 10, e0132436 (2015).

23. Rosen, D.B. et al. Functional consequences of interactions between human NKR-P1A and its ligand LLT1 expressed on activated dendritic cells and B cells. Journal of immunology 180, 6508-6517 (2008).

24. Boles, K.S., Barten, R., Kumaresan, P.R., Trowsdale, J. \& Mathew, P.A. Cloning of a new lectin-like receptor expressed on human NK cells. Immunogenetics 50, 1-7 (1999).

25. Roth, P. et al. Malignant glioma cells counteract antitumor immune responses through expression of lectin-like transcript-1. Cancer research 67, 3540-3544 (2007).

26. Mathew, S.O., Chaudhary, P., Powers, S.B., Vishwanatha, J.K. \& Mathew, P.A. Overexpression of LLT1 (OCIL, CLEC2D) on prostate cancer cells inhibits NK cellmediated killing through LLT1-NKRP1A (CD161) interaction. Oncotarget 7, 68650-68661 (2016).

27. Marrufo, A.M. et al. Blocking LLT1 (CLEC2D, OCIL)-NKRP1A (CD161) interaction enhances natural killer cell-mediated lysis of triple-negative breast cancer cells. Am J Cancer Res 8, 1050-1063 (2018).

28. Germain, C. et al. Lectin-like transcript 1 is a marker of germinal center-derived B-cell non-Hodgkin's lymphomas dampening natural killer cell functions. Oncoimmunology 4, e1026503 (2015).

29. Zambrano-Zaragoza, J.F., Romo-Martinez, E.J., Duran-Avelar Mde, J., GarciaMagallanes, N. \& Vibanco-Perez, N. Th17 cells in autoimmune and infectious diseases. International journal of inflammation 2014, 651503 (2014). 
30. Afzali, B. et al. CD161 expression characterizes a subpopulation of human regulatory T cells that produces IL-17 in a STAT3-dependent manner. European journal of immunology 43, 2043-2054 (2013).

31. Billerbeck, E. et al. Analysis of CD161 expression on human CD8+ T cells defines a distinct functional subset with tissue-homing properties. Proceedings of the National Academy of Sciences of the United States of America 107, 3006-3011 (2010).

32. Smith, J.A. \& Colbert, R.A. Review: The interleukin-23/interleukin-17 axis in spondyloarthritis pathogenesis: Th17 and beyond. Arthritis \& rheumatology 66, 231-241 (2014).

33. Brucklacher-Waldert, V., Stuerner, K., Kolster, M., Wolthausen, J. \& Tolosa, E. Phenotypical and functional characterization of $\mathrm{T}$ helper 17 cells in multiple sclerosis. Brain : a journal of neurology 132, 3329-3341 (2009).

34. Estrada-Capetillo, L. et al. Induction of Th17 lymphocytes and Treg cells by monocytederived dendritic cells in patients with rheumatoid arthritis and systemic lupus erythematosus. Clinical \& developmental immunology 2013, 584303 (2013).

35. Michalak-Stoma, A. et al. Serum levels of selected Th17 and Th22 cytokines in psoriatic patients. Disease markers 35, 625-631 (2013).

36. Germain, C. et al. Characterization of alternatively spliced transcript variants of CLEC2D gene. The Journal of biological chemistry 285, 36207-36215 (2010).

37. Li, Y., Wang, Q., Chen, S., Brown, P.H. \& Mariuzza, R.A. Structure of NKp65 bound to its keratinocyte ligand reveals basis for genetically linked recognition in natural killer gene complex. Proceedings of the National Academy of Sciences of the United States of America 110, 11505-11510 (2013).

38. Bauer, B., Spreu, J., Rohe, C., Vogler, I. \& Steinle, A. Key residues at the membranedistal surface of KACL, but not glycosylation, determine the functional interaction of the keratinocyte-specific C-type lectin-like receptor $\mathrm{KACL}$ with its high-affinity receptor NKp65. Immunology 145, 114-123 (2015).

39. Kamishikiryo, J., Fukuhara, H., Okabe, Y., Kuroki, K. \& Maenaka, K. Molecular basis for LLT1 protein recognition by human CD161 protein (NKRP1A/KLRB1). The Journal of biological chemistry 286, 23823-23830 (2011).

40. Kita, S. et al. Crystal structure of extracellular domain of human lectin-like transcript 1 (LLT1), the ligand for natural killer receptor-P1A. European journal of immunology 45, 1605-1613 (2015). 
41. Aguilar, O.A. et al. A Viral Immunoevasin Controls Innate Immunity by Targeting the Prototypical Natural Killer Cell Receptor Family. Cell 169, 58-71 e14 (2017).

42. Balaji, G.R. et al. Recognition of host Clr-b by the inhibitory NKR-P1B receptor provides a basis for missing-self recognition. Nat Commun 9, 4623 (2018).

43. Skalova, T. et al. Four crystal structures of human LLT1, a ligand of human NKR-P1, in varied glycosylation and oligomerization states. Acta crystallographica. Section D, Biological crystallography 71, 578-591 (2015).

44. Blaha, J., Pachl, P., Novak, P. \& Vanek, O. Expression and purification of soluble and stable ectodomain of natural killer cell receptor LLT1 through high-density transfection of suspension adapted HEK293S GnTI(-) cells. Protein expression and purification 109, 713 (2015).

45. Vanek, O. et al. Soluble recombinant CD69 receptors optimized to have an exceptional physical and chemical stability display prolonged circulation and remain intact in the blood of mice. The FEBS journal 275, 5589-5606 (2008).

46. Kolenko, P. et al. The high-resolution structure of the extracellular domain of human CD69 using a novel polymer. Acta Crystallogr Sect F Struct Biol Cryst Commun 65, 1258-1260 (2009).

47. Skalova, T. et al. Mouse Clr-g, a ligand for NK cell activation receptor NKR-P1F: crystal structure and biophysical properties. Journal of immunology 189, 4881-4889 (2012).

48. Lovell, S.C. et al. Structure validation by Calpha geometry: phi,psi and Cbeta deviation. Proteins 50, 437-450 (2003).

49. Brown, J. et al. Structure of the fungal beta-glucan-binding immune receptor dectin-1: implications for function. Protein science : a publication of the Protein Society 16, $1042-$ 1052 (2007).

50. Vanek, O. et al. Production of recombinant soluble dimeric C-type lectin-like receptors of rat natural killer cells. Scientific reports 9, 17836 (2019).

51. Blaha, J. et al. High-level expression and purification of soluble form of human natural killer cell receptor NKR-P1 in HEK293S GnTI- cells. Protein expression and purification 140, 36-43 (2017).

52. Rother, S. et al. The c.503T>C Polymorphism in the Human KLRB1 Gene Alters Ligand Binding and Inhibitory Potential of CD161 Molecules. PloS one 10, e0135682 (2015).

53. Skorepa, O. et al. Natural Killer Cell Activation Receptor NKp30 Oligomerization Depends on Its N-Glycosylation. Cancers (Basel) 12 (2020). 
54. Davis, D.M. et al. The human natural killer cell immune synapse. Proceedings of the National Academy of Sciences of the United States of America 96, 15062-15067 (1999).

55. Pageon, S.V. et al. Superresolution microscopy reveals nanometer-scale reorganization of inhibitory natural killer cell receptors upon activation of NKG2D. Science signaling 6 , ra62 (2013).

56. Stamper, C.C. et al. Crystal structure of the B7-1/CTLA-4 complex that inhibits human immune responses. Nature 410, 608-611 (2001).

57. Schwartz, J.C., Zhang, X., Fedorov, A.A., Nathenson, S.G. \& Almo, S.C. Structural basis for co-stimulation by the human CTLA-4/B7-2 complex. Nature 410, 604-608 (2001).

58. Bhatia, S., Sun, K., Almo, S.C., Nathenson, S.G. \& Hodes, R.J. Dynamic equilibrium of B7-1 dimers and monomers differentially affects immunological synapse formation and $T$ cell activation in response to TCR/CD28 stimulation. Journal of immunology 184, 18211828 (2010).

59. Reeves, P.J., Callewaert, N., Contreras, R. \& Khorana, H.G. Structure and function in rhodopsin: high-level expression of rhodopsin with restricted and homogeneous $\mathrm{N}$ glycosylation by a tetracycline-inducible $\mathrm{N}$-acetylglucosaminyltransferase I-negative HEK293S stable mammalian cell line. Proceedings of the National Academy of Sciences of the United States of America 99, 13419-13424 (2002).

60. Grueninger-Leitch, F., D'Arcy, A., D'Arcy, B. \& Chene, C. Deglycosylation of proteins for crystallization using recombinant fusion protein glycosidases. Protein science : a publication of the Protein Society 5, 2617-2622 (1996).

61. Kabsch, W. Xds. Acta crystallographica. Section D, Biological crystallography 66, 125132 (2010).

62. Evans, P.R. \& Murshudov, G.N. How good are my data and what is the resolution? Acta crystallographica. Section D, Biological crystallography 69, 1204-1214 (2013).

63. Long, F., Vagin, A.A., Young, P. \& Murshudov, G.N. BALBES: a molecular-replacement pipeline. Acta crystallographica. Section D, Biological crystallography 64, 125-132 (2008).

64. Li, Y. et al. Structure of natural killer cell receptor KLRG1 bound to E-cadherin reveals basis for MHC-independent missing self recognition. Immunity 31, 35-46 (2009).

65. McCoy, A.J. et al. Phaser crystallographic software. Journal of applied crystallography 40, 658-674 (2007). 
66. Kolenko, P. et al. Molecular architecture of mouse activating NKR-P1 receptors. Journal of structural biology 175, 434-441 (2011).

67. Vagin, A. \& Teplyakov, A. Molecular replacement with MOLREP. Acta crystallographica. Section D, Biological crystallography 66, 22-25 (2010).

68. Murshudov, G.N. et al. REFMAC5 for the refinement of macromolecular crystal structures. Acta crystallographica. Section D, Biological crystallography 67, 355-367 (2011).

69. Emsley, P., Lohkamp, B., Scott, W.G. \& Cowtan, K. Features and development of Coot. Acta crystallographica. Section D, Biological crystallography 66, 486-501 (2010).

70. Rozbesky, D. et al. High-level expression of soluble form of mouse natural killer cell receptor NKR-P1C(B6) in Escherichia coli. Protein expression and purification 77, 178184 (2011).

71. Schuck, P. Size-distribution analysis of macromolecules by sedimentation velocity ultracentrifugation and Lamm equation modeling. Biophysical journal 78, 1606-1619 (2000).

72. Brautigam, C.A. Calculations and Publication-Quality Illustrations for Analytical Ultracentrifugation Data. Methods in enzymology 562, 109-133 (2015).

73. Schuck, P. On the analysis of protein self-association by sedimentation velocity analytical ultracentrifugation. Analytical biochemistry 320, 104-124 (2003).

74. Scheuermann, T.H., Padrick, S.B., Gardner, K.H. \& Brautigam, C.A. On the acquisition and analysis of microscale thermophoresis data. Analytical biochemistry 496, 79-93 (2016).

75. Petoukhov, M.V. et al. New developments in the program package for small-angle scattering data analysis. Journal of applied crystallography 45, 342-350 (2012).

76. Franke, D. \& Svergun, D.I. DAMMIF, a program for rapid ab-initio shape determination in small-angle scattering. Journal of applied crystallography 42, 342-346 (2009).

77. Svergun, D.I. Restoring low resolution structure of biological macromolecules from solution scattering using simulated annealing. Biophysical journal 76, 2879-2886 (1999).

78. Pettersen, E.F. et al. UCSF Chimera--a visualization system for exploratory research and analysis. Journal of computational chemistry 25, 1605-1612 (2004). 
79. Konarev, P.V., Volkov, V.V., Skolova, A.V., Koch, M.H.J. \& Svergun, D.I. PRIMUS: a Windows PC-based system for small-angle scattering data analysis. Journal of applied crystallography $36,1277-1282$ (2003).

80. Li, Z., Michael, I.P., Zhou, D., Nagy, A. \& Rini, J.M. Simple piggyBac transposon-based mammalian cell expression system for inducible protein production. Proceedings of the National Academy of Sciences of the United States of America 110, 5004-5009 (2013).

81. Ovesny, M., Krizek, P., Borkovec, J., Svindrych, Z. \& Hagen, G.M. ThunderSTORM: a comprehensive ImageJ plug-in for PALM and STORM data analysis and superresolution imaging. Bioinformatics 30, 2389-2390 (2014).

82. Schneider, C.A., Rasband, W.S. \& Eliceiri, K.W. NIH Image to ImageJ: 25 years of image analysis. Nature methods 9, 671-675 (2012).

83. Thompson, R.E., Larson, D.R. \& Webb, W.W. Precise nanometer localization analysis for individual fluorescent probes. Biophysical journal 82, 2775-2783 (2002).

84. Andronov, L., Orlov, I., Lutz, Y., Vonesch, J.L. \& Klaholz, B.P. ClusterViSu, a method for clustering of protein complexes by Voronoi tessellation in super-resolution microscopy. Scientific reports 6, 24084 (2016). 